TRANSACTIONS OF THE

AMERICAN MATHEMATICAL SOCIETY

Volume 362, Number 7, July 2010, Pages 3613-3640

S 0002-9947(10)05044-0

Article electronically published on February 15, 2010

\title{
REAL PALEY-WIENER THEOREMS AND LOCAL SPECTRAL RADIUS FORMULAS
}

\author{
NILS BYRIAL ANDERSEN AND MARCEL DE JEU
}

\begin{abstract}
We systematically develop real Paley-Wiener theory for the Fourier transform on $\mathbb{R}^{d}$ for Schwartz functions, $L^{p}$-functions and distributions, in an elementary treatment based on the inversion theorem. As an application, we show how versions of classical Paley-Wiener theorems can be derived from the real ones via an approach which does not involve domain shifting and which may be put to good use for other transforms of Fourier type as well. An explanation is also given as to why the easily applied classical Paley-Wiener theorems are unlikely to be able to yield information about the support of a function or distribution which is more precise than giving its convex hull, whereas real Paley-Wiener theorems can be used to reconstruct the support precisely, albeit at the cost of combinatorial complexity. We indicate a possible application of real Paley-Wiener theory to partial differential equations in this vein, and furthermore we give evidence that a number of real PaleyWiener results can be expected to have an interpretation as local spectral radius formulas. A comprehensive overview of the literature on real PaleyWiener theory is included.
\end{abstract}

\section{INTRODUCTION AND OVERVIEW}

A Paley-Wiener theorem is a characterization, by relating support to growth, of the image of a space of functions or distributions under a transform of Fourier type. Starting with the original Paley-Wiener theorem, [48, Theorem X], which describes the Fourier transform of $L^{2}$-functions on the real line with support in a symmetric interval as entire functions of exponential type whose restriction to the real line are $L^{2}$-functions, such results have proven to be a basic tool for transform in various set-ups. As a familiar example, if $f$ is a smooth compactly supported function on $\mathbb{R}^{d}$ with Fourier transform $\mathcal{F} f: \mathbb{R}^{d} \rightarrow \mathbb{C}$, then it is easily seen that $\mathcal{F} f$ extends to an entire function on $\mathbb{C}^{d}$ with the property that, for each $n=0,1,2, \ldots$, there exists a constant $C_{n}$ such that

$$
|\mathcal{F} f(z)| \leq C_{n}(1+|z|)^{-n} e^{H_{A}(\operatorname{Im} z)} \quad\left(z \in \mathbb{C}^{d}\right),
$$

where $A$ is the convex hull of the support of $f$ and $H_{A}: \mathbb{R}^{d} \rightarrow \mathbb{R}$ is its supporting function, defined in terms of the standard inner product as $H_{A}(x)=\max _{a \in A} a \cdot x$,

Received by the editors May 12, 2008.

2010 Mathematics Subject Classification. Primary 42B10; Secondary 47A11.

Key words and phrases. Paley-Wiener theorem, Fourier transform, constant coefficient differential operator, local spectrum, local spectral radius formula.

The first author was supported by a research grant from the European Commission IHP Network: 2002-2006 Harmonic Analysis and Related Problems (Contract Number: HPRN-CT-200100273 - HARP).

(C)2010 American Mathematical Society Reverts to public domain 28 years from publication 
for $x \in \mathbb{R}^{d}$. The non-trivial part of the Paley-Wiener theorem for smooth functions is the converse: if $A$ is a compact and convex subset of $\mathbb{R}^{d}$, and if an entire function $g: \mathbb{C}^{d} \rightarrow \mathbb{C}$ satisfies estimates as in (1.1), then $g$ is the Fourier transform of a smooth function supported in $A$. Whereas this is all common knowledge, it seems to be less widely known that $H_{\operatorname{co}(\operatorname{supp} f)}$ - where $\operatorname{co}(\operatorname{supp} f)$ is the convex hull of the support of $f$-does not just give a theoretical upper bound for the dominant part of the growth of $\mathcal{F} f$ on $\mathbb{C}^{d}$ as in (1.1), but actually prescribes it: the exponential function in (1.1) is really needed, and precisely in this form. This observation goes back to Plancherel and Pólya [54; there is also a detailed account available in [56. Theorem 3.4.2]. To be more concrete, their result, when combined with [56, Theorem 3.4.3], implies that, if $y \in \mathbb{R}^{d}$ is fixed,

$$
\limsup _{t \rightarrow \infty} \frac{1}{t} \log |\mathcal{F} f(x+i t y)|=H_{\operatorname{co}(\operatorname{supp} f)}(y),
$$

for almost all $x$ in $\mathbb{R}^{d}$. Consequently, if $f_{1}$ and $f_{2}$ are smooth compactly supported functions such that the convex hulls of their supports are equal, and if $y \in \mathbb{R}^{d}$ is fixed, then $\lim \sup _{t \rightarrow \infty} \frac{1}{t} \log \left|\mathcal{F} f_{1}(x+i t y)\right|=\lim \sup _{t \rightarrow \infty} \frac{1}{t} \log \left|\mathcal{F} f_{2}(x+i t y)\right|$ for almost all $x$ in $\mathbb{R}^{d}$. Informally speaking, this means that the growth of $\mathcal{F} f$ on $\mathbb{C}^{d}$ enables one to retrieve the convex hull of the support of $f$, but that more precise information cannot readily be obtained from it. Hence the Paley-Wiener theorem is optimal in this respect. This phenomenon is not limited to smooth functions: [54] was already concerned with the more general $L^{2}$-case, and it is known [57, Theorem 5.1.2], 46, that the straightforward analogue of (1.2) is true for a general compactly supported distribution $T$. Thus the dominant part of the growth of $\mathcal{F} T$ on $\mathbb{C}^{d}$ is prescribed by the convex hull of the support of $T$, irrespective of the precise support or the degree of regularity of $T$, and the Paley-Wiener theorem for distributions with compact support is again optimal in this respect.

Whereas in view of the above there seems to exist a fundamental theoretical obstruction for the classical Paley-Wiener theorems to "look inside" the convex hull of the support, this is not the case for a new type of theorem which has enjoyed increasing interest in recent years. They have become known as "real Paley-Wiener theorems", in which the adjective "real" expresses that information about the support of $f$ comes from growth rates associated to the function $\mathcal{F} f$ on $\mathbb{R}^{d}$ rather than on $\mathbb{C}^{d}$ as in the classical "complex Paley-Wiener theorems". A typical example is the following: If $P$ is a polynomial with corresponding constant coefficient differential operator $P(\partial), f$ is a Schwartz function on $\mathbb{R}^{d}$, and $1 \leq p \leq$ $\infty$, then in the extended positive real numbers,

$$
\lim _{n \rightarrow \infty}\left\|P(\partial)^{n} f\right\|_{p}^{1 / n}=\sup _{\lambda \in \operatorname{supp} \mathcal{F} f}|P(i \lambda)| .
$$

Thus, as compared to the complex theorems, the real theorems involve a growth rate as $n \rightarrow \infty$ rather than $|z| \rightarrow \infty 1$ What is more important from a theoretical point of view is that if an upper bound $M$ for the left hand side in (1.3) is known, then one concludes that $\operatorname{supp} \mathcal{F} f$ is contained in $\left\{\lambda \in \mathbb{R}^{d}:|P(i \lambda)| \leq M\right\}$, which

\footnotetext{
${ }^{1}$ The roles of $f$ and $\mathcal{F} f$ in the literature on real Paley-Wiener theorems $\mathbb{R}^{d}$ are reversed as compared to the complex theorems so that some mental gymnastics is inevitable, but this is not a consequence of unfortunate choices. Both roles have their place in general Lie theory, and it is only because $\mathbb{R}^{d}$ happens to be self-dual that the results for the group and the unitary dual intermingle at equal footing. See also [18].
} 
need be neither compact nor convex. In fact, the complex theorem will not even apply if $\mathcal{F} f$ does not have compact support, although the real theorem still does. It is in this fashion that more information about the support can be extracted compared to using the complex theorems, albeit at the cost of combinatorial complexity. One can even reconstruct the support itself in a number of cases. As an example, it is one of our results that, if $1 \leq p \leq \infty, f$ is an $L^{p}$-function and $K$ is compact, then the support of the distribution $\mathcal{F} f$ is contained in $K$ if, and only if,

$$
\lim _{n \rightarrow \infty}\left\|P(\partial)^{n} f\right\|_{p}^{1 / n} \leq \sup _{k \in K}|P(i k)|,
$$

for all polynomials $P 2$ In Section 2, it is also explained how it sometimes is sufficient for this reconstruction to control $\left\|P(\partial)^{n} f\right\|_{p}^{1 / n}$ for sufficiently many polynomials, rather than for all of them. We have similar results for general tempered distributions.

Our results in real Paley-Wiener theory are contained in Section 2 below. We consider pointwise estimates as well as $L^{p}$-norms; Schwartz functions as well as $L^{p}$-functions and distributions; and single polynomial results such as (1.3) as well as multiple polynomial results such as (1.4). The reader who consults Bang's work, [19, 27, and Tuan's work, 70, 71, will see that our work is related to these papers, but that, where there is an overlap, our results are more general and the proofs are quite different. As an example, (1.3) for Schwartz functions was first established by Bang [19, in one dimension and with $P(x)=x$, using the complex Paley-Wiener theorem, and later by Tuan, in arbitrary dimension for real polynomials, using the Plancherel theorem [71. In our paper we systematically base our proofs on the inversion theorem, and this yields (1.3) for arbitrary polynomials, as well as its analogue for $L^{p}$-functions which was hitherto not within reach. Our proofs are also considerably more elementary, especially compared to the proofs using Sobolev theory for elliptic equations in [27. By giving a systematic broad development of the theory, based on simple proofs and extending as well as generalizing previous insights, we hope to provide a basis for future developments - also for other transforms - of this theory, which was initiated by Bang and Tuan.

It is noteworthy that real Paley-Wiener theorems can provide a new and alternative method of proof for complex Paley-Wiener theorems. This is illustrated in Section 3 below, where we derive complex Paley-Wiener theorems for the Fourier transform from the real ones in Section 2. One does not obtain the strongest possible forms of the complex theorems for the Fourier transform in this way, but the important feature of this approach is that it does not involve shifting of the domain, as in the usual proofs of the complex results. This may work for other integral transforms as well, and, in fact, in 17 it was demonstrated how this idea can be employed fruitfully to prove a complex Paley-Wiener theorem for the Dunkl transform in one dimension, where the idea of contour shifting simply does not apply since the integrand is not entire.

We have included a comprehensive overview of the literature on real PaleyWiener theory in Section 4 in which we discuss the most important contributions by Bang, Tuan and others, and compare them to our results. This section contains a fair number of references, and, although we do not claim completeness, we expect

\footnotetext{
${ }^{2}$ It follows from the hypotheses that $P(\partial)^{n} f$ is in $L^{p}$ for all $n$.
} 
that the material in this section will be useful to anyone who wants to gain an overview of the field at this time.

The final section, Section 5, contains perspectives for future developments. One of these, and an intriguing one in our opinion, is a possible interpretation of equations such as (1.3) in local spectral theory. Namely, it may well be that the right hand side of (1.3) is the radius of a local spectrum, so that (1.3) is a manifestation of a local spectral radius formula for an unbounded operator. For $p=1$ we are, in fact, able to prove this, and we have established similar results for compact connected Lie groups [18. Since we are not aware of general results on the a priori validity of a local spectral radius formula for unbounded operators (quite contrary to the bounded case), and since this also gives a new angle on real Paley-Wiener theorems, we discuss this subject in some detail. We are indebted to Jan van Neerven for suggesting the possibility of a connection with local spectral theory.

Notation and preliminaries. Our notations are the usual ones, as in [40] or [58, Chapter 7], where the proofs of the properties mentioned below can also be found. We let $\mathbb{N}=\{1,2,3, \ldots\}$ and $\mathbb{N}_{0}=\{0,1,2, \ldots\}$. Furthermore, $\mathcal{S}\left(\mathbb{R}^{d}\right)$ stands for the Schwartz space on $\mathbb{R}^{d}$ with dual $\mathcal{S}^{\prime}\left(\mathbb{R}^{d}\right)$, the space of tempered distributions on $\mathbb{R}^{d}$. Recall that $L^{p}\left(\mathbb{R}^{d}\right) \subset \mathcal{S}^{\prime}\left(\mathbb{R}^{d}\right)$, for all $1 \leq p \leq \infty$. If $P$ is a polynomial on $\mathbb{R}^{d}$, we let $P(\partial)$ denote the corresponding differential operator with constant coefficients, so that $P(\partial) e^{i \lambda \cdot x}=P(i \lambda)^{i \lambda \cdot x}$, for all $\lambda, x$. For $T \in \mathcal{S}^{\prime}\left(\mathbb{R}^{d}\right)$, the distribution $P(\partial) T \in$ $\mathcal{S}^{\prime}\left(\mathbb{R}^{d}\right)$ is defined by

$$
\langle P(\partial) f, \phi\rangle=\langle f, P(-\partial) \phi\rangle \quad\left(\phi \in \mathcal{S}\left(\mathbb{R}^{d}\right)\right),
$$

which is compatible with the action on smooth functions. The convolution $f * g$ of two functions $f, g \in L^{1}\left(\mathbb{R}^{d}\right)$ is defined as

$$
f * g(x)=\int_{\mathbb{R}^{d}} f(x-y) g(y) d y \quad\left(x \in \mathbb{R}^{d}\right) .
$$

If $f \in L^{p}\left(\mathbb{R}^{d}\right), 1 \leq p \leq \infty$, and $g \in L^{1}\left(\mathbb{R}^{d}\right)$, then the integral (1.5) converges for almost all $x \in \mathbb{R}^{d}$, and we have the following inequality:

$$
\|f * g\|_{p} \leq\|f\|_{p}\|g\|_{1} .
$$

We also notice that

$$
P(\partial)(f * \phi)=f * P(\partial) \phi,
$$

for all $f \in L^{p}\left(\mathbb{R}^{d}\right), 1 \leq p \leq \infty$, and $\phi \in \mathcal{S}\left(\mathbb{R}^{d}\right)$.

Our normalization of the Fourier transform $\mathcal{F} f$ of a function $f \in L^{1}\left(\mathbb{R}^{d}\right)$ is as

$$
\mathcal{F} f(\lambda)=\frac{1}{(2 \pi)^{d / 2}} \int_{\mathbb{R}^{d}} f(x) e^{-i \lambda \cdot x} d x \quad\left(\lambda \in \mathbb{R}^{d}\right),
$$

where $\lambda \cdot x=\sum_{i=1}^{d} \lambda_{i} x_{i}$. The inversion formula then holds with the same constant $(2 \pi)^{-d / 2}$, and the Plancherel theorem is valid with Lebesgue measure by both sides. The Fourier transform $\mathcal{F} T$ of a tempered distribution $T$ is defined

$$
\langle\mathcal{F} T, \phi\rangle=\langle T, \mathcal{F} \phi\rangle \quad\left(\phi \in \mathcal{S}\left(\mathbb{R}^{d}\right)\right),
$$

which is compatible with its definition on $L^{1}\left(\mathbb{R}^{d}\right)$. Then, for all $T \in \mathcal{S}^{\prime}\left(\mathbb{R}^{d}\right)$,

$$
\mathcal{F}(P(\partial) T)(\lambda)=P(i \lambda) \mathcal{F} T(\lambda) \quad(\lambda \in \mathbb{R}) .
$$

Also,

$$
\mathcal{F}(f * \phi)=\mathcal{F} f \cdot \mathcal{F} \phi \quad\left(f \in L^{p}\left(\mathbb{R}^{d}\right), \phi \in \mathcal{S}\left(\mathbb{R}^{d}\right)\right) .
$$


It is with some emphasis (see the discussion in Section 4 on the preference for proofs based on the inversion theorem over those based on the Plancherel theorem) that we mention that all polynomials are complex valued unless otherwise stated.

\section{Real Paley-Wiener theorems}

Let $f$ be a measurable function on $\mathbb{R}^{d}$ representing a tempered distribution, and let $P$ be a polynomial. For $f$ in various classes, this section is concerned with the relation between the growth behavior of the sequence $\left\{P(\partial)^{n} f\right\}_{n=0}^{\infty}$ on $\mathbb{R}^{d}$ and the supremum of $|P(i \lambda)|$ on the support of $\mathcal{F} f$. The notation for quantities such as the latter is introduced in the following definition.

Definition 2.1. Let $T$ be a distribution on $\mathbb{R}^{d}$ and $P$ a polynomial. Then we let

$$
R(P, T)=\sup \{|P(i \lambda)|: \lambda \in \operatorname{supp} T\} \in \mathbb{R}_{\geq 0} \cup\{\infty\},
$$

where by convention $R(P, T)=0$ if $T=0$.

A typical statement in the sequel will be that $R(P, \mathcal{F} f) \leq R$, for some $R \geq 0$, or, equivalently, that $\operatorname{supp} \mathcal{F} f \subset\left\{\lambda \in \mathbb{R}^{d}:|P(i \lambda)| \leq R\right\}$. We will also be concerned with reconstructing $\operatorname{supp} \mathcal{F} f$, given the knowledge that it is in sufficiently many of such polynomially defined sets.

Our first result in this vein is the following real Paley-Wiener theorem for Schwartz functions. The corresponding results for tempered distributions in general are included as Propositions 2.10 and 2.11, and Theorem 2.12.

Theorem 2.2. Let $P$ be a polynomial, $f \in \mathcal{S}\left(\mathbb{R}^{d}\right)$, and $0<R<\infty$. Then the following are equivalent:

(a) There exists a function $\phi: \mathbb{N} \rightarrow \mathbb{R}_{+}$such that

$$
\liminf _{n \rightarrow \infty} \phi(n)^{1 / n} \geq 1 / R
$$

and

$$
\sup _{n \in \mathbb{N}} \phi(n)\left\|(1+|x|)^{d+1} P(\partial)^{n} f\right\|_{\infty}<\infty .
$$

(b) For each $N \in \mathbb{N}_{0}$, there exists a constant $C$ such that, for all $n \in \mathbb{N}$ and $x \in \mathbb{R}^{d}$,

$$
\left|P(\partial)^{n} f(x)\right| \leq C n^{N} R^{n}(1+|x|)^{-N} .
$$

(c) $R(P, \mathcal{F} f) \leq R$.

Proof. First we prove that (a) implies (c). Assume that $\left|P\left(i \lambda_{0}\right)\right| \geq R+\varepsilon$ for some $\lambda_{0} \in \mathbb{R}^{d}$ and $\varepsilon>0$. The implication will follow once we show that $\mathcal{F} f\left(\lambda_{0}\right)=0$. For $n \in \mathbb{N}$, one has

$$
\mathcal{F} f\left(\lambda_{0}\right)=\frac{1}{(2 \pi)^{d / 2} P\left(i \lambda_{0}\right)^{n}} \int_{\mathbb{R}^{d}}\left(P(\partial)^{n} f\right)(x) e^{-i \lambda_{0} \cdot x} d x .
$$

Hence, for all $n \in \mathbb{N}$ large enough (so that $\phi(n) \neq 0$ ),

$$
\begin{aligned}
\left|\mathcal{F} f\left(\lambda_{0}\right)\right| & \leq \frac{1}{(2 \pi)^{d / 2}\left|P\left(i \lambda_{0}\right)\right|^{n}} \int_{\mathbb{R}^{d}}(1+|x|)^{-d-1}\left|(1+|x|)^{d+1}\left(P(\partial)^{n} f\right)(x)\right| d x \\
& \leq C \phi(n)^{-1}\left|P\left(i \lambda_{0}\right)\right|^{-n}
\end{aligned}
$$

for some positive constant $C$. So

$$
\left|\mathcal{F} f\left(\lambda_{0}\right)\right| \leq C \limsup _{n \rightarrow \infty}\left(\phi(n)^{-1}\left|P\left(i \lambda_{0}\right)\right|^{-n}\right) \leq C \limsup _{n \rightarrow \infty} \frac{(R+\varepsilon / 2)^{n}}{(R+\varepsilon)^{n}}=0 .
$$


Now we prove that (c) implies that

$$
\sup _{n \in \mathbb{N}} R^{-n} n^{-N}\left\||x|^{N} P(\partial) f\right\|_{\infty}<\infty
$$

for each $N \in \mathbb{N}_{0}$, which is easily seen to be equivalent to (b). In order to establish (2.1), we will first show that

$$
\sup _{n \in \mathbb{N}} R^{-n} n^{-N}\left\|(y \cdot x)^{N} P(\partial)^{n} f\right\|_{\infty}<\infty,
$$

for each $N \in \mathbb{N}_{0}$ and $y \in \mathbb{R}^{d}$ (where the supremum norm is taken of the function of $x$ ). Fix such $N$ and $y$. Then, for all $n \in \mathbb{N}$ and all $x \in \mathbb{R}^{d}$, we have

$$
\begin{aligned}
(y \cdot x)^{N} P(\partial)^{n} f(x) & =\frac{i^{N}}{(2 \pi)^{d / 2}} \int_{\mathbb{R}^{d}} \partial_{y}^{N}\left[P(i \lambda)^{n} \mathcal{F} f(\lambda)\right] e^{i \lambda \cdot x} d \lambda \\
& =\frac{i^{N}}{(2 \pi)^{d / 2}} \int_{\mathbb{R}^{d}}\left(\sum_{k=0}^{N}\left(\begin{array}{c}
N \\
k
\end{array}\right) \partial_{y}^{k} P(i \lambda)^{n} \partial_{y}^{N-k} \mathcal{F} f(\lambda)\right) e^{i \lambda \cdot x} d \lambda .
\end{aligned}
$$

An induction with respect to $k$ shows that

$$
\begin{aligned}
\partial_{y}^{k} P(i \lambda)^{n} & =\sum_{l=0}^{k} n(n-1) \cdots(n-l+1) P_{l, k}(\lambda) P(i \lambda)^{n-l} \\
& =P(i \lambda)^{n-k} \sum_{l=0}^{k} n(n-1) \cdots(n-l+1) P_{l, k}(\lambda) P(i \lambda)^{k-l},
\end{aligned}
$$

for some polynomials $P_{l, k}$ independent of $n$. Here we have assumed that $n>N$, so that $n-k>0$ for all $k$ that occur in the summation; hence $P(i \lambda)^{n-k}$ is defined. This implies that, for $k$ occurring in the summation, $n>N$, and $\lambda \in \sup \mathcal{F} f$,

$$
\begin{aligned}
\left|\partial_{y}^{k} P(i \lambda)^{n}\right| & \leq R(P, \mathcal{F} f)^{n-k} n^{N} \sum_{l=0}^{k}\left|P_{l, k}(\lambda) P(i \lambda)^{k-l}\right| \\
& \leq R^{n} n^{N} \sum_{l=0}^{k} R^{-k}\left|P_{l, k}(\lambda) P(i \lambda)^{k-l}\right|,
\end{aligned}
$$

and using this in (2.3) shows that (2.2) holds with the supremum taken over $n>N$, which implies (2.2) itself.

To conclude the proof that (c) implies (b), we choose a basis $y_{1}, \ldots, y_{d}$ of $\mathbb{R}^{d}$. Then there exists $C>0$ such that $|x| \leq C \max _{j}\left|y_{j} \cdot x\right|$, for all $x \in \mathbb{R}^{d}$; hence $|x|^{N} \leq C^{N} \sum_{j=1}^{d}\left|y_{j} \cdot x\right|^{N}$. Using this and applying (2.2) to the $y_{j}$ shows that (2.1) holds.

Finally, to prove that (b) implies (a), we simply take $\phi(n)=R^{-n} n^{-d-1}$.

Remark 2.3. Controlling $P(\partial)^{n} f$ as in (a) or (b) implies that $\operatorname{supp} \mathcal{F} f \subseteq\{\lambda \in$ $\left.\mathbb{R}^{d}:|P(i \lambda)| \leq R\right\}$. As the example $P\left(x_{1}, x_{2}\right)=x_{1} x_{2}$ on $\mathbb{R}^{2}$ shows, this latter set can be non-compact and non-convex. This illustrates the point, mentioned in the Introduction, that the theoretical limitations to compact and convex sets which seem to exist for complex Paley-Wiener theorems are not present in the real case.

We will now study the $L^{p}$-case, for which we need the following key proposition. 
Proposition 2.4. Let $P$ be a polynomial and $1 \leq p \leq \infty$. Suppose $P(\partial)^{n} f \in$ $L^{p}\left(\mathbb{R}^{d}\right)$, for all $n \in \mathbb{N}_{0}$. Then in the extended positive real numbers,

$$
\liminf _{n \rightarrow \infty}\left\|P(\partial)^{n} f\right\|_{p}^{1 / n} \geq R(P, \mathcal{F} f) .
$$

Proof. Fix $\lambda_{0} \in \operatorname{supp} \mathcal{F} f$. We can assume that $P\left(i \lambda_{0}\right) \neq 0$. We will show that

$$
\liminf _{n \rightarrow \infty}\left\|P(\partial)^{n} f\right\|_{p}^{1 / n} \geq\left|P\left(i \lambda_{0}\right)\right|-\varepsilon,
$$

for any (fixed) $\varepsilon>0$ such that $0<2 \varepsilon<\left|P\left(i \lambda_{0}\right)\right|$.

To this end, choose and fix $\psi \in C_{c}^{\infty}\left(\mathbb{R}^{d}\right)$ such that $\langle\mathcal{F} f, \psi\rangle \neq 0$ and

$$
\operatorname{supp} \psi \subset\left\{\lambda \in \mathbb{R}^{d}:\left|P\left(i \lambda_{0}\right)\right|-\varepsilon<|P(i \lambda)|<\left|P\left(i \lambda_{0}\right)\right|+\varepsilon\right\} .
$$

This is possible since $\lambda_{0}$ belongs to the set in (2.6) and $\lambda_{0} \in \operatorname{supp} \mathcal{F} f$. For $n \in \mathbb{N}_{0}$, let $\psi_{n}(\lambda)=P(i \lambda)^{-n} \psi(\lambda)$. Let $\frac{1}{p}+\frac{1}{q}=1$, and fix $M \in \mathbb{N}$ such that $\left(1+|x|^{2}\right)^{-M} \in$ $L^{q}\left(\mathbb{R}^{d}\right)$. We note that

$$
\left(1+|x|^{2}\right)^{M} \mathcal{F} \psi_{n}(x)=\frac{1}{(2 \pi)^{d / 2}} \int_{\mathbb{R}^{d}}(1-\Delta)^{M}\left\{P(i \lambda)^{-n} \psi(\lambda)\right\} e^{-i \lambda \cdot x} d \lambda,
$$

where $\Delta$ is the Laplacian. When expanding $(1-\Delta)^{M}\left\{P(i \lambda)^{-n} \psi(\lambda)\right\}$, it can be written as a finite sum

$$
\sum_{\alpha, \beta} c_{\alpha, \beta}\left(D^{\alpha}\left(P(i \lambda)^{-n}\right)\right)\left(D^{\beta} \psi\right)
$$

for coefficients $c_{\alpha, \beta}$ and composites $D^{\alpha}, D^{\beta}$ of partial derivatives of order $|\alpha|,|\beta| \leq$ $2 M$. Neither the coefficients nor the differential operators depend on $n$.

Let $n>2 M$. Using induction with respect to $|\alpha|$, it is seen that, if $P(i \lambda) \neq 0$,

$$
\begin{aligned}
D^{\alpha}\left(P(i \lambda)^{-n}\right) & =\sum_{k=0}^{|\alpha|} n(n+1) \cdots(n+k-1) P_{k, \alpha}(\lambda) P(i \lambda)^{-n-k} \\
& =P(i \lambda)^{-n-|\alpha|} \sum_{k=0}^{|\alpha|} n(n+1) \cdots(n+k-1) P_{k, \alpha}(\lambda) P(i \lambda)^{|\alpha|-k},
\end{aligned}
$$

where the $P_{k, \alpha}$ are polynomials independent of $n$. For all $\alpha$ occurring in the expansion one has $|\alpha| \leq 2 M$; hence for each occurring $\alpha$ there exists a positive $C_{\alpha}$ such that

$$
\left|D^{\alpha}\left(P(i \lambda)^{-n}\right)\right| \leq C_{\alpha} n^{2 M}\left(\left|P\left(i \lambda_{0}\right)\right|-\varepsilon\right)^{-n-|\alpha|},
$$

for all $n>2 M$ and all $\lambda \in \operatorname{supp} \psi$. Therefore there exists a positive $C_{1}$ such that

$$
\left|(1-\Delta)^{M}\left\{P(i \lambda)^{-n} \psi(\lambda)\right\}\right| \leq C_{1} n^{2 M}\left(\left|P\left(i \lambda_{0}\right)\right|-\varepsilon\right)^{-n},
$$

for all $n>2 M$ and all $\lambda \in \mathbb{R}^{d}$. Using this in (2.7), we conclude that there exists a positive constant $C_{2}$ such that

$$
\left|\left(1+|x|^{2}\right)^{M} \mathcal{F} \psi_{n}(x)\right| \leq C_{2} n^{2 M}\left(\left|P\left(i \lambda_{0}\right)\right|-\varepsilon\right)^{-n},
$$

for all $n>2 M$ and $x \in \mathbb{R}^{d}$. It follows from this that there exists a positive constant $C_{3}$ such that

$$
\left\|\mathcal{F} \psi_{n}\right\|_{q} \leq C_{3} n^{2 M}\left(\left|P\left(i \lambda_{0}\right)\right|-\varepsilon\right)^{-n},
$$

for all $n>2 M$. Then, since

$$
\langle\mathcal{F} f, \psi\rangle=\left\langle\mathcal{F} f, P(i \lambda)^{n} \psi_{n}\right\rangle=\left\langle P(i \lambda)^{n} \mathcal{F} f, \psi_{n}\right\rangle=\left\langle\mathcal{F}\left(P(\partial)^{n} f\right), \psi_{n}\right\rangle=\left\langle P(\partial)^{n} f, \mathcal{F} \psi_{n}\right\rangle,
$$


by (1.6) and (1.7), we use the assumption that $P(\partial)^{n} f \in L^{p}\left(\mathbb{R}^{d}\right)$, for all $n \in \mathbb{N}_{0}$, and Hölder's inequality to obtain

$$
|\langle\mathcal{F} f, \psi\rangle| \leq\left\|P(\partial)^{n} f\right\|_{p}\left\|\mathcal{F} \psi_{n}\right\|_{q} \leq C_{4} n^{2 M}\left(\left|P\left(i \lambda_{0}\right)\right|-\varepsilon\right)^{-n}\|(P(\partial)) f\|_{p},
$$

for all $n>2 M$. Since $|\langle\mathcal{F} f, \psi\rangle|>0$, we conclude that (2.5) holds.

Combining Proposition 2.4 above with Theorem 2.2 yields two results in an $L^{p_{-}}$ context. In Section 5 we will comment on their possible interpretation in local spectral theory. The first result is for Schwartz functions.

Theorem 2.5. Let $P$ be a polynomial, $1 \leq p \leq \infty$, and $f \in \mathcal{S}\left(\mathbb{R}^{d}\right)$. Then in the extended positive real numbers

$$
\lim _{n \rightarrow \infty}\left\|P(\partial)^{n} f\right\|_{p}^{1 / n}=R(P, \mathcal{F} f) .
$$

Proof. In view of Proposition 2.4 it is sufficient to prove that in the extended positive real numbers

$$
\limsup _{n \rightarrow \infty}\left\|P(\partial)^{n} f\right\|_{p}^{1 / n} \leq R(P, \mathcal{F} f)
$$

and for this we may, and will, assume that $0<R(P, \mathcal{F} f)<\infty$. We fix $M \in \mathbb{N}$ such that $(1+|x|)^{-M} \in L^{p}\left(\mathbb{R}^{d}\right)$. From Theorem 2.2, we see that there exists a positive constant $C$ such that

$$
\left\|(1+|x|)^{M} P(\partial)^{n} f\right\|_{\infty} \leq C n^{M} R(P, \mathcal{F} f)^{n},
$$

for all $n \in \mathbb{N}$, which implies that

$$
\left\|P(\partial)^{n} f\right\|_{p} \leq\left\|(1+|x|)^{-M}\right\|_{p}\left\|(1+|x|)^{N} P(\partial)^{n} f\right\|_{\infty} \leq C^{\prime} n^{M} R(P, \mathcal{F} f)^{n},
$$

for a new positive constant $C^{\prime}$. This completes the proof.

The second consequence of the combination of Proposition 2.4 and Theorem 2.2 is valid for a class of $L^{p}$-functions. There is overlap with the previous Theorem 2.5. but neither statement contains the other as a special case.

Theorem 2.6. Let $P$ be a polynomial and $1 \leq p \leq \infty$. Suppose $P(\partial)^{n} f \in L^{p}\left(\mathbb{R}^{d}\right)$, for all $n \in \mathbb{N}_{0}$. Assume furthermore that either $\mathcal{F} f$ has compact support or that the set $\left\{\lambda \in \mathbb{R}^{d}:|P(i \lambda)| \leq R\right\}$ is compact for all $R \geq 0$. Then in the extended positive real numbers

$$
\lim _{n \rightarrow \infty}\left\|P(\partial)^{n} f\right\|_{p}^{1 / n}=R(P, \mathcal{F} f) .
$$

Proof. From Proposition 2.4, we have

$$
\liminf _{n \rightarrow \infty}\left\|P(\partial)^{n} f\right\|_{p}^{1 / n} \geq R(P, \mathcal{F} f) .
$$

As to the first case, assume that $\mathcal{F} f$ has compact support. Fix $\varepsilon>0$. We will prove that

$$
\limsup _{n \rightarrow \infty}\left\|P(\partial)^{n} f\right\|_{p}^{1 / n} \leq R(P, \mathcal{F} f)+\varepsilon .
$$

To this end, we choose a $\psi \in \mathcal{S}\left(\mathbb{R}^{d}\right)$ such that $\mathcal{F} \psi=1$ on supp $\mathcal{F} f$ and $|P(i \lambda)|<$ $R(P, \mathcal{F} f)+\varepsilon$, for all $\lambda \in \operatorname{supp} \mathcal{F} \psi$. In particular, $R(P, \mathcal{F} \psi)<R(P, \mathcal{F} f)+\varepsilon$. By the compactness of $\operatorname{supp} \mathcal{F} f$, this is indeed possible. Since $\mathcal{F}(f * \psi)=\mathcal{F} f \cdot \mathcal{F} \psi=\mathcal{F} f$, we see that $f * \psi=f$; hence

$$
\left\|P(\partial)^{n} f\right\|_{p}=\left\|P(\partial)^{n}(f * \psi)\right\|_{p}=\left\|f * P(\partial)^{n} \psi\right\|_{p} \leq\|f\|_{p}\left\|P(\partial)^{n} \psi\right\|_{1} .
$$


Then, as a consequence of Theorem 2.5 (or, given the proof of that result, actually already as a consequence of Theorem [2.2),

$$
\limsup _{n \rightarrow \infty}\left\|P(\partial)^{n} f\right\|_{p}^{1 / n} \leq \limsup _{n \rightarrow \infty}\left\|P(\partial)^{n} \psi\right\|_{1}^{1 / n} \leq R(P, \mathcal{F} \psi)<R(P, \mathcal{F} f)+\varepsilon,
$$

as desired.

As to the second case, assume that the set $\left\{\lambda \in \mathbb{R}^{d}:|P(i \lambda)| \leq R\right\}$ is compact for all $R>0$. If $R(P, \mathcal{F} f)<\infty$, then $\mathcal{F} f$ has compact support, and the result follows from the first case. If $R(P, \mathcal{F} f)=\infty$, the result follows from Proposition 2.4

In particular, if one assumes that $\Delta^{n} f \in L^{p}\left(\mathbb{R}^{d}\right)$, for all $n \in \mathbb{N}_{0}$, and $0 \leq R<\infty$, then $\lim _{n \rightarrow \infty}\left\|\Delta^{n} f\right\|_{p}^{1 / n} \leq R$ if, and only if, $\mathcal{F} f$ has support in the ball of radius $R$ around the origin.

For $p=2$, we can remove the compactness restrictions in Theorem 2.6 by using the Plancherel theorem. This is one of the two applications of the Plancherel theorem in the present paper, the other one being in the proof of Theorem 3.5

Theorem 2.7. Let $P$ be a polynomial, and suppose $P(\partial)^{n} f \in L^{2}\left(\mathbb{R}^{d}\right)$, for all $n \in \mathbb{N}_{0}$. Then in the extended positive real numbers

$$
\lim _{n \rightarrow \infty}\left\|P(\partial)^{n} f\right\|_{2}^{1 / n}=R(P, \mathcal{F} f) .
$$

Proof. By Proposition 2.4, we only need to show that

$$
\limsup _{n \rightarrow \infty}\left\|P(\partial)^{n} f\right\|_{2}^{1 / n} \leq R(P, \mathcal{F} f) .
$$

We may assume that $R(P, \mathcal{F} f)<\infty$. For all $\psi \in \mathcal{S}\left(\mathbb{R}^{d}\right)$, we have

$$
\left\langle P(\partial)^{n} f, \psi\right\rangle=\left\langle P(\partial)^{n} f, \mathcal{F} \mathcal{F}^{-1} \psi\right\rangle=\left\langle\mathcal{F}\left(P(\partial)^{n} f\right), \mathcal{F}^{-1} \psi\right\rangle=\left\langle P(i \lambda)^{n} \mathcal{F} f, \mathcal{F}^{-1} \psi\right\rangle,
$$

so using the Plancherel theorem in the final step we obtain that

$$
\begin{aligned}
\left|\left\langle P(\partial)^{n} f, \psi\right\rangle\right| & =\left|\int_{\mathbb{R}^{d}} P(i \lambda)^{n}(\mathcal{F} f(\lambda))\left(\mathcal{F}^{-1} \psi(\lambda)\right) d \lambda\right| \\
& \leq R(P, \mathcal{F} f)^{n} \int_{\mathbb{R}^{d}}\left|\mathcal{F} f(\lambda) \mathcal{F}^{-1} \psi(\lambda)\right| d \lambda \\
& \leq R(P, \mathcal{F} f)^{n}\|\mathcal{F} f\|_{2}\left\|\mathcal{F}^{-1} \psi\right\|_{2} \\
& =R(P, \mathcal{F} f)^{n}\|f\|_{2}\|\psi\|_{2} .
\end{aligned}
$$

Since

$$
\left\|P(\partial)^{n} f\right\|_{2}=\sup _{\psi \in \mathcal{S}\left(\mathbb{R}^{d}\right),\|\psi\|_{2}=1}\left|\left\langle P(\partial)^{n} f, \psi\right\rangle\right|,
$$

we conclude that

$$
\left\|P(\partial)^{n} f\right\|_{2} \leq R(P, \mathcal{F} f)^{n}\|f\|_{2},
$$

and (2.13) follows.

If the Fourier transform $\mathcal{F} f$ of a function $f \in L^{p}\left(\mathbb{R}^{d}\right)$ is compact, then controlling $\left\|P(\partial)^{n} f\right\|_{p}$ for sufficiently many polynomials $P$ enables one to find the precise support of $\mathcal{F} f$. This is expressed by part (c) and (d) of Theorem 2.9 below, a key ingredient for which is the following. 
Lemma 2.8. Let $K$ be a non-empty compact subset of $\mathbb{R}^{d}$. Then there exists a set $\mathcal{P}_{K}$ of polynomials such that

$$
K=\bigcap_{P \in \mathcal{P}_{K}}\left\{\lambda \in \mathbb{R}^{d}:|P(i \lambda)| \leq \max _{\lambda \in K}|P(i \lambda)|\right\} .
$$

Indeed, as a consequence of the Stone-Weierstraß theorem, taking the set of all polynomials for $\mathcal{P}_{K}$ certainly works. However, depending on the geometry of $K-$ on which one may sometimes have a priori information - much smaller sets may be sufficient. For a ball, a single polynomial of degree two will do, and for convex hulls of finitely many points, finitely many polynomials of degree one suffice. It is with this in mind that part (c) in Theorem 2.9 and part (b) in Theorem 2.15 should be read.

Theorem 2.9. Let $1 \leq p \leq \infty$, and assume the Fourier transform $\mathcal{F} f$ of $f \in$ $L^{p}\left(\mathbb{R}^{d}\right)$ has compact support. Let $K$ be a non-empty compact subset of $\mathbb{R}^{d}$. Then

(a) $P(\partial) f$ is in $L^{p}\left(\mathbb{R}^{d}\right)$, for all polynomials $P$.

(b) For any set $\mathcal{P}_{K}$ determining $K$ as in Lemma 2.8, supp $\mathcal{F} f \subset K$ if, and only if,

$$
\lim _{n \rightarrow \infty}\left\|P(\partial)^{n} f\right\|_{p}^{1 / n} \leq \max _{\lambda \in K}|P(i \lambda)|,
$$

for all $P$ in $\mathcal{P}_{K}$.

(c) For any set $\mathcal{P}_{\text {supp } \mathcal{F} f}$ determining $\operatorname{supp} \mathcal{F} f$ as in Lemma 2.8, $\lambda \in \mathbb{R}^{d}$ is in $\operatorname{supp} \mathcal{F} f$ if, and only if,

$$
|P(i \lambda)| \leq \lim _{n \rightarrow \infty}\left\|P(\partial)^{n} f\right\|_{p}^{1 / n},
$$

for all $P \in \mathcal{P}_{\text {supp }} \mathcal{F} f$.

(d) One can reconstruct $\operatorname{supp} \mathcal{F} f$ as

$$
\operatorname{supp} \mathcal{F} f=\left\{\lambda \in \mathbb{R}^{d}:|P(i \lambda)| \leq \lim _{n \rightarrow \infty}\left\|P(\partial)^{n} f\right\|_{p}^{1 / n} \text {, for all polynomials } P\right\} .
$$

The existence of the finite limits in (b) and (c) is guaranteed by (a) and Theorem 2.6 .

Proof. Choose $\psi \in C_{c}^{\infty}\left(\mathbb{R}^{d}\right)$ which is equal to 1 on $\operatorname{supp} \mathcal{F} f$. If $P$ is a polynomial, then, as distributions, $P(\partial) f=f * P(\partial) \mathcal{F}^{-1} \psi$, as can be seen by taking Fourier transforms and using [62, Theorem 30.4]. Since $L_{1}$ acts on $L_{p}$ by convolution, (a) follows. Theorem 2.6 gives (b) and (c). Since we already noted that the set of all polynomials will always do for any compact set, (d) follows from (c).

We now turn to general tempered distributions.

Proposition 2.10. Let $T$ be a distribution of order $N$ with compact support, and let $P$ be a polynomial. Then, for each $R>R(P, T)$, there exists a constant $C$, such that, for all $n \in \mathbb{N}$ and $x \in \mathbb{R}^{d}$,

$$
\left|P(\partial)^{n} \mathcal{F}^{-1} T(x)\right| \leq C n^{N} R^{n}(1+|x|)^{N} .
$$

Proof. Let $V_{R}=\left\{\lambda \in \mathbb{R}^{d}:|P(i \lambda)| \leq R\right\}$. Then $V_{R}$ contains the open neighborhood $\left\{\lambda \in \mathbb{R}^{d}:|P(i \lambda)|<R\right\}$ of $\operatorname{supp} T$. Hence we can choose $\psi \in C_{c}^{\infty}\left(\mathbb{R}^{d}\right)$ such that 
$\psi=1$ on an open neighborhood of $\operatorname{supp} T$ and $\psi=0$ outside $V_{R}$. Since $T$ is of order $N$, there exists a constant $C^{\prime}$, such that, for all $x_{0} \in \mathbb{R}^{d}$,

$$
\begin{aligned}
\left|\left(P(\partial)^{n} \mathcal{F}^{-1} T\right)\left(x_{0}\right)\right| & =\left|\mathcal{F}^{-1}\left(P(i \lambda)^{n} T\right)\left(x_{0}\right)\right| \\
& =\left|\mathcal{F}^{-1}\left(\psi P(i \lambda)^{n} T\right)\left(x_{0}\right)\right| \\
& =\left|\left\langle\psi P(i \lambda)^{n} T, e^{i x_{0} \cdot \lambda}\right\rangle\right| \\
& =\left|\left\langle T, \psi P(i \lambda)^{n} e^{i x_{0} \cdot \lambda}\right\rangle\right| \\
& \leq C^{\prime} \sum_{|\alpha| \leq N}\left\|D^{\alpha}\left(\psi P(i \lambda)^{n} e^{i x_{0} \cdot \lambda}\right)\right\|_{\infty} .
\end{aligned}
$$

Induction with respect to $|\alpha|$ shows that

$$
D^{\alpha}\left(\psi P(i \lambda)^{n} e^{i x_{0} \cdot \lambda}\right)=\sum_{k=0}^{|\alpha|} n(n-1) \cdots(n-k+1) \psi_{k, \alpha}(\lambda) Q_{k, \alpha}\left(x_{0}\right) e^{i x_{0} \cdot \lambda} P(i \lambda)^{n-k},
$$

where the $\psi_{k, \alpha}$ are smooth functions, independent of $n$ and $x_{0}$, and vanishing outside $\operatorname{supp} \psi$, and the $Q_{k, \alpha}$ are polynomials, independent of $n$ and $\lambda$, and of degree at most $|\alpha|$. For $n>N$, this can be written, for all $\lambda \in \mathbb{R}^{d}$, as

$$
P(i \lambda)^{n-N} \sum_{k=0}^{|\alpha|} n(n-1) \cdots(n-k+1) \psi_{k, \alpha}(\lambda) Q_{k, \alpha}\left(x_{0}\right) e^{i x_{0} \cdot \lambda} P(i \lambda)^{N-k} .
$$

The vanishing property of the $\psi_{k, \alpha}$ therefore implies that the supremum norm of this function is bounded by

$$
c_{\alpha} R^{n} n^{|\alpha|}\left(1+\left|x_{0}\right|\right)^{|\alpha|}
$$

for some constant $c_{\alpha}$. Hence there exists a constant $C$ such that the inequality in the theorem holds for $n>N$, and increasing $C$ if necessary yields the desired inequality for all $n \in \mathbb{N}$.

As to the reverse implication, we have the following result.

Proposition 2.11. Let $f \in C^{\infty}\left(\mathbb{R}^{d}\right)$ be a tempered distribution, and suppose there exist a polynomial $P$, an integer $N \in \mathbb{N}_{0}$, and constants $C, R \geq 0$, such that, for all $n \in \mathbb{N}$ and $x \in \mathbb{R}^{d}$,

$$
\left|P(\partial)^{n} f(x)\right| \leq C n^{N} R^{n}(1+|x|)^{N} .
$$

Then $R(P, \mathcal{F} f) \leq R$.

Note that it is not assumed that $\operatorname{supp} \mathcal{F} f$ is compact.

Proof. Suppose $\lambda_{0} \in \mathbb{R}^{d}$ is fixed and such that $\left|P\left(i \lambda_{0}\right)\right| \geq R+\varepsilon$, for some $\varepsilon>0$. Let $V=\left\{\lambda \in \mathbb{R}^{d}:|P(i \lambda)|>R+\varepsilon / 2\right\}$, and suppose $\psi \in C_{c}^{\infty}\left(\mathbb{R}^{d}\right)$ is supported in $V$. We will show that $\langle\mathcal{F} f, \psi\rangle=0$, and hence $\lambda_{0} \notin \operatorname{supp} \mathcal{F} f$, which implies the theorem.

To this end we introduce, for $n \in \mathbb{N}$, the function $\psi_{n}(\lambda)=\psi(\lambda) P(i \lambda)^{-n}$, which is a well defined compactly supported smooth function. If $M \in \mathbb{N}$ is any fixed integer such that $\left(1+|x|^{2}\right)^{-M} \in L^{1}\left(\mathbb{R}^{d}\right)$, then as in the proof of Proposition 2.4 one concludes that there is a constant $C^{\prime} \geq 0$ such that, for all $x \in \mathbb{R}^{d}$ and all $n>2 M$,

$$
\left(1+|x|^{2}\right)^{M}\left|\mathcal{F} \psi_{n}(x)\right| \leq C^{\prime} n^{2 M}\left(R+\frac{\varepsilon}{2}\right)^{-n} .
$$


Hence, if we choose $M \in \mathbb{N}$ such that $\left(1+|x|^{2}\right)^{-M}(1+|x|)^{N} \in L^{1}\left(\mathbb{R}^{d}\right)$, then, for $n>2 M$ and $x \in \mathbb{R}^{d}$,

$$
\begin{aligned}
|\langle\mathcal{F} f, \psi\rangle| & =\left|\left\langle f, \mathcal{F}\left(P(i \lambda)^{n} \psi_{n}\right)\right\rangle\right| \\
& =\left|\left\langle f, P(-\partial)^{n} \mathcal{F} \psi_{n}\right\rangle\right| \\
& =\left|\left\langle P(\partial)^{n} f, \mathcal{F} \psi_{n}\right\rangle\right| \\
& =\left|\int_{\mathbb{R}^{d}} P(\partial)^{n} f(x)\left(1+|x|^{2}\right)^{-M}\left(1+|x|^{2}\right)^{M} \mathcal{F} \psi_{n}(x) d x\right| \\
& \leq \int_{\mathbb{R}^{d}} C n^{N} R^{n}(1+|x|)^{N}\left(1+|x|^{2}\right)^{-M} C^{\prime} n^{2 M}\left(R+\frac{\varepsilon}{2}\right)^{-n} d x \\
& \leq C^{\prime \prime} n^{N+2 M}\left(\frac{R}{R+\frac{\varepsilon}{2}}\right)^{n},
\end{aligned}
$$

and the theorem follows.

In view of the proof of Theorem 3.7 below, we note that the proof of Proposition 2.11 above shows that it is sufficient that (2.14) holds for all but finitely many $n$.

Combining Proposition 2.10 and a special case of Proposition 2.11 yields the following characterization of distributions with compact support, as a real counterpart of the classical complex result.

Theorem 2.12. Let $f \in C^{\infty}\left(\mathbb{R}^{d}\right)$ be a tempered distribution, and suppose the set $\left\{\lambda \in \mathbb{R}^{d}:|P(i \lambda)| \leq R_{0}\right\}$ is compact for a polynomial $P$ and a constant $R_{0} \geq 0$. Then the support of $\mathcal{F} f$ is contained in $\left\{\lambda \in \mathbb{R}^{d}:|P(i \lambda)| \leq R_{0}\right\}$ if, and only if, for each $R>R_{0}$, there exist constants $N_{R} \in \mathbb{N}_{0}$ and $C_{R} \geq 0$ such that

$$
\left|P(\partial)^{n} f(x)\right| \leq C_{R} n^{N_{R}} R^{n}(1+|x|)^{N_{R}},
$$

for all $n \in \mathbb{N}$ and $x \in \mathbb{R}^{d}$. If this is the case, then, for all $R>R_{0}$, one can in fact take $N_{R}$ equal to the order of $\mathcal{F} f$ in (2.15).

Theorems 2.6 and 2.9 for $L^{p}$ also have an analogue for distributions with compact support, for which we need a definition.

Definition 2.13. Let $f \in C^{\infty}\left(\mathbb{R}^{d}\right)$ be a tempered distribution, such that $\operatorname{supp} \mathcal{F} f$ is compact. Let $P$ be a polynomial. Then we define $\widetilde{R}(P, f)$ as the infimum of all $R \geq 0$, for which there exist constants $N \in \mathbb{N}_{0}$ and $C_{N, R} \geq 0$ such that, for all $n \in \mathbb{N}$ and $x \in \mathbb{R}^{d}$,

$$
\left|P(\partial)^{n} f(x)\right| \leq C_{N, R} n^{N} R^{n}(1+|x|)^{N} .
$$

The analogue of Theorem 2.6 is then the following, with $\widetilde{R}(P, f)$ taking over the role of $\lim _{n \rightarrow \infty}\left\|P(\partial)^{n} f\right\|_{p}^{1 / n}$.

Theorem 2.14. Let $f \in C^{\infty}\left(\mathbb{R}^{d}\right)$ be a tempered distribution such that $\operatorname{supp} \mathcal{F} f$ is compact. Let $P$ be a polynomial. Then $\widetilde{R}(P, f)=R(P, \mathcal{F} f)$.

Proof. By Proposition 2.10, $\widetilde{R}(P, f) \leq R(P, \mathcal{F} f)$. The reverse inequality follows from Proposition 2.11

The reconstruction of supports, as in Theorem 2.9 for the $L^{p}$-case, now takes the following form. 
Theorem 2.15. Let $f \in C^{\infty}\left(\mathbb{R}^{d}\right)$ be a tempered distribution such that $\operatorname{supp} \mathcal{F} f$ is compact. Let $K$ be a non-empty compact subset of $\mathbb{R}^{d}$. Then

(a) For any set $\mathcal{P}_{K}$ determining $K$ as in Lemma 2.8, supp $\mathcal{F} f \subset K$ if, and only if,

$$
\widetilde{R}(P, f) \leq \max _{\lambda \in K}|P(i \lambda)|,
$$

for all $P$ in $\mathcal{P}_{K}$.

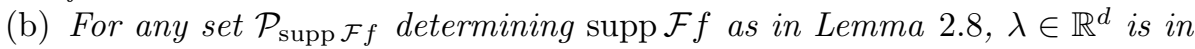
$\operatorname{supp} \mathcal{F} f$ if, and only if,

$$
|P(i \lambda)| \leq \widetilde{R}(P, f),
$$

for all $P \in \mathcal{P}_{\operatorname{supp}} \mathcal{F} f$.

(c) One can reconstruct $\operatorname{supp} \mathcal{F} f$ as

$$
\operatorname{supp} \mathcal{F} f=\left\{\lambda \in \mathbb{R}^{d}:|P(i \lambda)| \leq \widetilde{R}(P, f) \text {, for all polynomials } P\right\} \text {. }
$$

Proof. Part (a) follows from Proposition 2.10, as does part (b). Part (c) is a special case of part (b).

\section{Proving complex Paley-Wiener theorems WITHOUT DOMAIN SHIFTING}

As mentioned in the Introduction, there are instances where proving a complex Paley-Wiener theorem for an integral transform is not possible using domain shifting, since the integrand is not entire. In that case, results as in Section 2 may offer an alternative approach by deriving the complex theorems from the real ones, as is done in 17. To illustrate this point, we show how versions of a number of classical complex Paley-Wiener theorems for the Fourier transform follow from the real theorems in Section 2. The strategy is to apply the Cauchy formula to an entire function $f$, and then exploit the given usual estimates for $f$ on $\mathbb{C}^{d}$ to obtain upper bounds for $\left\|\partial_{\xi}^{n} f\right\|_{\infty}$ on $\mathbb{R}^{d}$, for all $n \in \mathbb{N}$ and $\xi \in \mathbb{R}^{d}$. Combining this with a special case of Proposition 2.4 gives upper bounds for $\max _{x \in \operatorname{supp}} \mathcal{F} f|\xi \cdot x|$, for all $\xi \in \mathbb{R}^{d}$, and then a basic separation theorem from convex analysis implies the desired statement concerning $\operatorname{supp} \mathcal{F} f$ or $\operatorname{supp} \mathcal{F}^{-1} f$.

While this approach could offer an alternative where domain shifting is not valid, as of yet it seems to be limited to supports contained in a compact convex symmetric set. The symmetry condition is not necessary for the validity of the statement in the Fourier case, but controlling $\left\|\partial_{\xi}^{n} f\right\|_{\infty}$ on $\mathbb{R}^{d}$ can never yield a result which is stronger than that $\operatorname{supp} \mathcal{F} f$ is contained in some symmetric set, simply because $\max _{x \in A}|\xi \cdot x|=\max _{x \in-A}|\xi \cdot x|$. As demonstrated by, e.g., Theorem [2.9] it is in principle possible to reconstruct $\operatorname{supp} \mathcal{F} f$ by controlling $\left\|P(\partial)^{n} f\right\|_{\infty}$ on $\mathbb{R}^{d}$ for sufficiently many polynomials $P$, so that one might hope to be able to infer the general complex case via the real Proposition 2.4 by invoking more polynomials than just the first degree homogeneous ones. The combinatorics that arise when trying to obtain estimates via the Cauchy formula for $\left\|P(\partial)^{n} f\right\|_{\infty}$ for more general $P$ seem to get rather involved though, and it remains to be seen whether a real approach to the full complex result is actually feasible.

For functions, the only result we will need from Section 2 is Proposition 2.4 for $p=\infty$ and homogeneous polynomials of degree one. If the function in Proposition 2.4 is a Schwartz function, then we can also use the following ad hoc result 
with a much simpler proof. We include it to illustrate that deriving the complex Theorem 3.4 via real results is rather elementary.

Lemma 3.1. Let $P$ be a homogeneous polynomial with real coefficients, $f \in \mathcal{S}\left(\mathbb{R}^{d}\right)$, and $1 \leq p \leq \infty$. Then in the extended positive real numbers

$$
\liminf _{n \rightarrow \infty}\left\|P(\partial)^{n} f\right\|_{p}^{1 / n} \geq R(P, \mathcal{F} f) .
$$

Obviously the result remains true for polynomials which are a scalar multiple of a polynomial as in the lemma.

Proof. Let $\lambda_{0} \in \operatorname{supp} \mathcal{F} f$, and assume $P\left(i \lambda_{0}\right) \neq 0$. We choose and fix an $\varepsilon>0$ such that $0<\varepsilon<\left|P\left(i \lambda_{0}\right)\right|$. We will show that

$$
\liminf _{n \rightarrow \infty}\left\|P(\partial)^{n} f\right\|_{p}^{1 / n} \geq\left|P\left(i \lambda_{0}\right)\right|-\varepsilon,
$$

which will establish (3.1). Define, for $j \in\{0,1,2,3\}$,

$$
\psi_{j}(\lambda)=\overline{\mathcal{F}\left(P(\partial)^{j} f\right)(\lambda)} \quad\left(\lambda \in \mathbb{R}^{d}\right) .
$$

Then, using (1.6) and (1.7), Hölder's inequality yields

$$
\begin{aligned}
\left\|P(\partial)^{4 n+j} f\right\|_{p}\left\|\mathcal{F} \psi_{j}\right\|_{q} \\
\quad \geq\left|\left\langle P(\partial)^{4 n+j} f, \mathcal{F} \psi_{j}\right\rangle\right| \\
\quad=\left|\left\langle\mathcal{F}\left(P(\partial)^{4 n+j} f\right), \psi_{j}\right\rangle\right| \\
\quad=\left|\int_{\mathbb{R}^{d}} P(i \lambda)^{4 n} \mathcal{F}\left(P(\partial)^{j} f\right)(\lambda) \psi_{j}(\lambda) d \lambda\right| \\
\quad=\int_{\mathbb{R}^{d}}|P(i \lambda)|^{4 n}\left|\mathcal{F}\left(P(\partial)^{j} f\right)\right|^{2} d \lambda \\
\quad \geq\left(\left|P\left(i \lambda_{0}\right)\right|-\varepsilon\right)^{4 n} \int_{\left\{\lambda:|P(i \lambda)| \geq\left|P\left(i \lambda_{0}\right)\right|-\varepsilon\right\}}\left|P(i \lambda)^{2 j}\right||\mathcal{F} f(\lambda)|^{2} d \lambda .
\end{aligned}
$$

As the integral in the last line is strictly positive due to the fact that $\lambda_{0} \in \operatorname{supp} \mathcal{F} f$, this yields (3.2). Note that both assumptions on $P$ were used in passing from the fourth line to the fifth in the above display of equations.

Before passing to the complex Paley-Wiener theorems, let us establish terminology. If $A$ is a non-empty subset of $\mathbb{R}^{d}$, then we define the supporting function $H_{A}: \mathbb{R}^{d} \rightarrow \mathbb{R}^{d}$ of $A$ as $H_{A}(x)=\max _{a \in A} a \cdot x$, for $x \in \mathbb{R}^{d}$.

Definition 3.2. Let $A$ be a subset of $\mathbb{R}^{d}$, and let $f: \mathbb{C}^{d} \rightarrow \mathbb{C}$. We say that $f$ is an entire function on $\mathbb{C}^{d}$ of exponential type corresponding to $A$ if $f$ is entire and if there exists a positive constant $C$ such that

$$
|f(z)| \leq C e^{H_{A}(\operatorname{Im} z)} \quad\left(z \in \mathbb{C}^{d}\right) .
$$

We further say that $f$ is a rapidly decreasing entire function on $\mathbb{C}^{d}$ of exponential type corresponding to $A$ if $f$ is entire, and, for each $n \in \mathbb{N}_{0}$, there exists a positive constant $C_{n}$ such that

$$
|f(z)| \leq C_{n}(1+|z|)^{-n} e^{H_{A}(\operatorname{Im} z)} \quad\left(z \in \mathbb{C}^{d}\right) .
$$


As is well known, if an entire function $f$ satisfies (3.4), then, using the Cauchy formula, one sees that the partial derivatives $\partial_{\xi}^{n} f$ satisfy similar estimates, for all $\xi \in \mathbb{R}^{d}$ and $n \in \mathbb{N}$. Hence the restriction of $f$ to $\mathbb{R}^{d}$ is a Schwartz function, explaining the terminology.

The following result is the pivot in the transition from the complex to the real domain in the case of functions.

Lemma 3.3. Let $A$ be a non-empty compact symmetric subset of $\mathbb{R}^{d}$, and suppose $f$ is an entire function on $\mathbb{C}^{d}$ of exponential type corresponding to $A$. Then, for all $\xi \in \mathbb{R}^{d}$ and $n \in \mathbb{N}$, the partial derivative $\partial_{\xi}^{n} f$ is bounded on $\mathbb{R}^{d}$, and

$$
\limsup _{n \rightarrow \infty}\left\|\partial_{\xi}^{n} f\right\|_{\infty}^{1 / n} \leq H_{A}(\xi) \text {. }
$$

Proof. We claim that

$$
\left\|\partial_{\xi}^{n} f\right\|_{\infty} \leq C \frac{n ! e^{n}}{n^{n}}\left(H_{A}(\xi)\right)^{n}
$$

for $\xi \in \mathbb{R}^{d}$ and $n \in \mathbb{N}$, where the constant $C$ is as in (3.3). Indeed, Cauchy's theorem yields, for arbitrary $r>0$, that

$$
\partial_{\xi}^{n} f(x)=\left.\frac{d^{n}}{d t^{n}}\right|_{t=0}\{t \mapsto f(x+t \xi)\}=\frac{n !}{2 \pi i} \oint_{|t|=r} \frac{f(x+t \xi)}{t^{n+1}} d t \quad\left(x \in \mathbb{R}^{d}\right),
$$

and thus

$$
\left|\partial_{\xi}^{n} f(x)\right| \leq C \frac{n !}{r^{n}} e^{\max _{a \in A,|t|=r} a \cdot \operatorname{Im}(t \xi)}=C \frac{n !}{r^{n}} e^{r \max _{a \in A}|a \cdot \xi|}=C \frac{n !}{r^{n}} e^{r H_{A}(\xi)},
$$

where the symmetry of $A$ is used in the final equality. If $H_{A}(\xi)=0$, letting $r \rightarrow \infty$ shows that $\partial_{\xi}^{n} f=0$, for $n \in \mathbb{N}$, and (3.6) is proved. If $H_{A}(\xi) \neq 0$, then $H_{A}(\xi)>0$ by the symmetry of $A$; hence we can choose $r=n / H_{A}(\xi)$, and this again establishes (3.6). Then (3.5) follows from Stirling's formula.

We now use Lemmas 3.1 and 3.3 to prove the symmetric version of the complex Paley-Wiener theorem for smooth functions.

Theorem 3.4. Let $A$ be a non-empty compact, convex and symmetric subset of $\mathbb{R}^{d}$, and suppose $f: \mathbb{R}^{d} \rightarrow \mathbb{C}$. Then the following are equivalent:

(a) $f$ is the Fourier transform of a smooth function with support contained in A.

(b) $f$ extends to a rapidly decreasing entire function on $\mathbb{C}^{d}$ of exponential type corresponding to $A$.

Proof. It is classical and easy to see that (a) implies (b).

As to the converse, assume that $f$ is a rapidly decreasing entire function on $\mathbb{C}^{d}$ of exponential type corresponding to $A$. Suppose that $\lambda_{0} \in \operatorname{supp} \mathcal{F} f$, but $\lambda_{0} \notin A$. Then, since $A$ is convex and closed, and $\left\{\lambda_{0}\right\}$ is convex and compact, by a standard separation result there exists $\xi_{0} \in \mathbb{R}^{d}$ such that $\lambda_{0} \cdot \xi_{0}>H_{A}\left(\xi_{0}\right)$. Consider the polynomial $P_{\xi_{0}}(\lambda)=\lambda \cdot \xi_{0}$, for $\lambda \in \mathbb{R}^{d}$. By Lemma 3.1 and Lemma 3.3.

$$
\begin{aligned}
\sup _{\lambda \in \operatorname{supp} \mathcal{F} f}\left|\lambda \cdot \xi_{0}\right| & =\sup _{\lambda \in \operatorname{supp} \mathcal{F} f}\left|P_{\xi_{0}}(i \lambda)\right| \leq \liminf _{n \rightarrow \infty}\left\|\partial_{\xi_{0}}^{n} f\right\|_{\infty}^{1 / n} \\
& \leq \limsup _{n \rightarrow \infty}\left\|\partial_{\xi_{0}}^{n} f\right\|_{\infty}^{1 / n} \leq H_{A}\left(\xi_{0}\right),
\end{aligned}
$$


so that $H_{A}\left(\xi_{0}\right)<\lambda_{0} \cdot \xi_{0} \leq\left|\lambda_{0} \cdot \xi_{0}\right| \leq H_{A}\left(\xi_{0}\right)$, a contradiction. We conclude that $\mathcal{F} f$ has support in $A$. Since $\mathcal{F}^{-1} f(x)=\mathcal{F} f(-x)$, for $x \in \mathbb{R}^{d}$, the symmetry of $A$ implies that $\mathcal{F}^{-1} f$ also has support contained in $A$.

The complex symmetric Paley-Wiener theorem for $L^{2}$-functions follows in the same fashion, using Lemma 3.3 and Proposition 2.4, the latter as a stronger replacement of Lemma 3.1 in the proof of Theorem 3.4 above. The proof contains one of the two applications of the Plancherel theorem in the present paper, the other one being in the proof of Theorem 2.7.

Theorem 3.5. Let $A$ be a non-empty compact, convex and symmetric subset of $\mathbb{R}^{d}$, and suppose $f$ is a measurable function on $\mathbb{R}^{d}$ representing a tempered distribution. Then the following are equivalent:

(a) $f$ is the Fourier transform of a function in $L^{2}\left(\mathbb{R}^{d}\right)$ with support contained in $A$.

(b) $f$ is in $L^{2}\left(\mathbb{R}^{d}\right)$ and extends to an entire function on $\mathbb{C}^{d}$ of exponential type corresponding to $A$.

Proof. It is obvious that (a) implies (b). Assuming (b), the Plancherel theorem yields that $\mathcal{F}^{-1} f \in L^{2}\left(\mathbb{R}^{d}\right)$. Applying Lemma 3.3 and the case $p=\infty$ of Proposition 2.4 to $f$ yields that, for all $\xi \in \mathbb{R}^{d}$, $\sup _{x \in \operatorname{supp} \mathcal{F} f}|\xi \cdot x| \leq H_{A}(\xi)$. A separation argument as in the proof of Theorem 3.4 shows that $\operatorname{supp} \mathcal{F} f$ is contained in $A$, and then the symmetry of $A$ implies that the same is true for $\operatorname{supp} \mathcal{F}^{-1} f$.

Remark 3.6. Let $A$ be a non-empty compact, convex and symmetric subset of $\mathbb{R}^{d}$. Let $1 \leq p \leq \infty$, and define $\mathcal{H}_{A}^{p}\left(\mathbb{C}^{d}\right)$ as the space of entire functions $f$ of exponential type corresponding to $A$, whose restriction to $\mathbb{R}^{d}$ belongs to $L^{p}\left(\mathbb{R}^{d}\right)$. As in the proof of Theorem 3.5 above, applying Lemma 3.3 and the case $p=\infty$ of Proposition 2.4 to such $f$ yields that the distribution $\mathcal{F} f$ (and hence also $\mathcal{F}^{-1} f$ ) has support in $A$, for all $f \in \mathcal{H}_{A}^{p}\left(\mathbb{C}^{d}\right)$. In particular, for $1 \leq p \leq 2$ with conjugate exponent $q$, we have established by real methods that $\mathcal{F}$ and $\mathcal{F}^{-1}$ map $\mathcal{H}_{A}^{p}\left(\mathbb{C}^{d}\right)$ into a subspace of $L^{q}\left(\mathbb{R}^{d}\right)$ consisting of functions with distributional support in $A$.

The symmetric complex Paley-Wiener theorem for distributions with compact support can be derived using the real Proposition 2.11.

Theorem 3.7. Let $A$ be a non-empty compact, convex and symmetric subset of $\mathbb{R}^{d}$, and suppose $f: \mathbb{R}^{d} \rightarrow \mathbb{C}$. Then the following are equivalent:

(a) $f$ is the Fourier transform of a distribution with support contained in $A$.

(b) $f$ extends to a entire function on $\mathbb{C}^{d}$, and there exist an integer $N \in \mathbb{N}_{0}$ and a constant $C$ such that $|f(z)| \leq C(1+|z|)^{N} e^{H_{A}(\operatorname{Im} z)}$, for all $z \in \mathbb{C}^{d}$.

If this is the case, then the integer $N$ in (b) can be taken to be the order of $\mathcal{F}^{-1} f$.

Proof. We need only prove that (b) implies (a), the rest being obvious. Fix $\xi \in \mathbb{R}^{d}$. We claim that there exists a constant $C^{\prime}$ such that, for all $x \in \mathbb{R}^{d}$ and $n>N$,

$$
\left|\partial_{\xi}^{n} f(x)\right| \leq C^{\prime} n^{N+1} H_{A}(\xi)^{n}(1+|x|)^{N+1} .
$$

Once this has been established, we infer from Proposition 2.11 and the subsequent remark that, for all $\xi \in \mathbb{R}^{d}, \max _{\lambda \in \operatorname{supp}} \mathcal{F}^{-1} f|\lambda \cdot \xi| \leq H_{A}(\xi)$. Statement (a) then follows from a separation argument as in the proof of Theorem 3.4. As to (3.7), a 
contour integral as in the proof of Lemma 3.3 yields that, for all $x \in \mathbb{R}^{d}, n \in \mathbb{N}$, and $r>0$,

$$
\left|\partial_{\xi}^{n} f(x)\right| \leq C \frac{n !}{r^{n}}(1+|x|+r|\xi|)^{N} e^{r H_{A}(\xi)},
$$

where $C$ is as in (b). If $H_{A}(\xi)=0$, then, as $n>N$, letting $r \rightarrow \infty$ establishes (3.7). If $H_{A}(\xi) \neq 0$, taking $r=n / H_{A}(\xi)>0$ shows that, for all $x \in \mathbb{R}^{d}$ and $n \in \mathbb{N}$,

$$
\begin{aligned}
\left|\partial_{\xi}^{n} f(x)\right| & \leq C \frac{n ! e^{n}}{n^{n}} H_{A}(\xi)^{n}\left(1+|x|+\frac{n|\xi|}{H_{A}(\xi)}\right)^{N} \\
& \leq C n^{N} \frac{n ! e^{n}}{n^{n}} H_{A}(\xi)^{n}\left(1+|x|+\frac{|\xi|}{H_{A}(\xi)}\right)^{N},
\end{aligned}
$$

and then Stirling's formula yields (3.7).

\section{Literature REVIEW}

In Section 2, we have established real Paley-Wiener theorems for the Fourier transform, and in Section 3 these have been used to derive complex Paley-Wiener theorems without domain shifting. We will now describe the historical development of the field and compare our results with the literature.

In the late fifties and early sixties, the Russian school studied families of test function spaces on the real line satisfying certain duality relations under the Fourier transform, the so-called Gelfand-Shilov spaces; see [39, Chapter 4]. For example, the Gelfand-Shilov spaces $S_{\alpha}^{\beta}(\alpha, \beta \geq 0)$ of type $S$ consist of all smooth functions $f$ on $\mathbb{R}$ satisfying

$$
\left|x^{k} f^{(q)}(x)\right| \leq C A^{k} B^{q} k^{k \alpha} q^{q \beta} \quad\left(x \in \mathbb{R}, k \in \mathbb{N}_{0}, q \in \mathbb{N}_{0}\right),
$$

for constants $A, B$ and $C$ depending on the function $f$. We note that $\mathcal{F}\left(S_{\alpha}^{\beta}\right)=S_{\beta}^{\alpha}$ and that $S_{0}^{\beta}$ consists of functions with compact support. The growth estimates (4.1) are similar to the ones in Theorem 2.2(b), but are concerned with repeated application of two operators rather than one, as in our case, and the differential operator is always monomial. The $L^{p}$-norms for $1 \leq p<\infty$ are furthermore not considered in [39]. In spite of the difference with later work by other authors, some of the results on the Gelfand-Shilov spaces in 39] should probably be thought of as the first real Paley-Wiener type theorems.

In 1990, see [19], Bang studied the growth of derivatives of an $L^{p}$-function $f$ on the real line, and, assuming that all derivatives of $f$ are also $L^{p}$-functions, found the relation [19, Theorem 1]

$$
\lim _{n \rightarrow \infty}\left\|f^{(n)}\right\|_{p}^{1 / n}=\sup \{|\lambda|: \lambda \in \operatorname{supp} \mathcal{F} f\}
$$

between the support of the Fourier transform of $f$ and the growth of these derivatives. This is our Theorem 2.6 for $d=1$ and $P(x)=x$. We believe that (4.2) is the first result relating the $L^{p}$-norm of a repeated application of a differential operator to $f$ to the supremum of the modulus of the corresponding multiplier on the support of the transform of $f$, i.e., the subject proper of the present article. Bang's proof of (4.2) uses the Kolmogorov and Bernstein inequalities and the complex Paley-Wiener theorem. There is a simplified approach in [13].

Bang, on his own and with coauthors, has since generalized (4.2) to the Fourier transform in Euclidean space in higher dimensions, as well as to Orlicz spaces and Lorentz spaces (with the appropriate associated operators); see [20, 21], 22., 23], 
24, 25], 26, 27, 28, 29, 30], 31, 32, and 33. In [19, Bang also covers the case of $L^{p}$-functions on the one-dimensional torus for $P(x)=x$, again using a complex Paley-Wiener theorem.

An approach using the Plancherel formula and intertwining properties was suggested by Tuan in 1995; see 64 and 70. The philosophical approach to more general set-ups is given by [65] and [71, in particular [71, Theorem 1], which explains, also in higher dimensions, why the existence of such generalizations is plausible for $p=2$ in the presence of a Plancherel theorem, at least for functions in a suitable subspace of the $L^{2}$-space under consideration. The new approach made it possible for Tuan and various coworkers to generalize Bang's result to several other integral transforms, mostly defined via differential equations (Dirac, Sturm-Liouville, etc.) on the real line; see [6], [7, [8, 69, 66], 67, 68, 69, 72, 73, [74, 75, 76], 77. and [78].

From 2000 onwards, the methods by Bang and Tuan and the simplified approach from [13] have been used to find real Paley-Wiener type theorems for a number of integral transforms. We refer to [1, [10, 11, [12, [14, 15, 16, [17, 34, 35, [36, [41, 45], 47, 63. for more details. Finally, we mention results by Pesenson in a similar vein; see [49, 50, 51, 52, 53. Here, in an $L^{2}$-context, bandlimited functions, i.e., functions with a compactly supported Fourier transform, on various manifolds are described by generalized Bernstein inequalities, and applications to sampling theory are given.

We will now discuss the most important contributions by Bang and Tuan, and compare them with our results.

After the initial work in one dimension in [19, Bang considered $L^{p}$-functions in higher dimensions in 21, but still for monomial operators only. The main result is the following: Let $0<p \leq \infty, f \in L^{p}\left(\mathbb{R}^{d}\right)$, and assume that $\operatorname{supp} \mathcal{F} f$ is compact. Then

$$
\lim _{|\alpha| \rightarrow \infty}\left(\frac{\left\|\partial^{\alpha} f\right\|_{p}}{\sup _{\lambda \in \operatorname{supp} \mathcal{F} f}\left|\lambda^{\alpha}\right|}\right)^{1 /|\alpha|}=1,
$$

where $\alpha$ is a multi-index, and $\lambda^{\alpha}$ has the usual meaning. The proof is rather technical and uses, e.g., Sobolev theory for elliptic equations. It is further shown in 23] that one can let $p$ depend on $\alpha$ by choosing $1 \leq p_{\alpha} \leq \infty$ and still obtain similar results.

The step to arbitrary polynomial operators and also to distributions was taken by Bang in [27, who was not concerned with $L^{p}$-norms, but with pointwise estimates. Some of the proofs are again rather technical, using Sobolev theory for elliptic equations in combination with structure theory for distributions. One of the main results, [27, Theorem 1], which is established using these techniques, is as follows: Let $K$ be an arbitrary compact set in $\mathbb{R}^{d}$. Then a tempered distribution $T$ has support in $K$ if, and only if, $\mathcal{F} T$ is a smooth function with the property that there exists an integer $N \in \mathbb{N}_{0}$ such that, for every $\delta>0$, there exists a constant $C_{\delta}$ such that

$$
|P(i \partial) \mathcal{F} T(\lambda)| \leq C_{\delta}(1+|\lambda|)^{N} \sup _{z \in K_{(\delta)}}|P(z)| \quad\left(\lambda \in \mathbb{R}^{d}\right)
$$

for all polynomials $P$, where $K_{(\delta)}$ is defined as the $\delta$-neighborhood of $K$ in $\mathbb{C}^{d}$. There is a similar result, [27, Theorem 2], for Schwartz functions. While these two 
results certainly yield information about the support, they are of a different flavor than ours, since $\mathbb{C}^{d}$ is still involved and our results are entirely formulated on $\mathbb{R}^{d}$. Furthermore, such information can only be obtained once one controls the left hand side for all polynomials, with a universal constant on the right hand side. This may not always be feasible, and our single polynomial results, also giving non-compact information, can then be more practical.

The results in 27] which are closest to ours are Theorem 5 for compactly supported distributions and Theorem 6 for Schwartz functions. Theorem 5 follows from our Propositions 2.10 and 2.11] but its proof in 27] is much more involved. Conversely, Bang's Theorem 5 implies our Proposition 2.10, but not our Proposition 2.11, which has no compactness assumptions. Bang's Theorem 6 is equivalent to our Theorem 2.2. and has a proof in the same spirit as our proofs 3 Bang was also the first to give reconstructing theorems for the support of distributions and functions in special cases. He has results in two contexts: using monomials [27, Theorems 3,4] and using polynomials of a given maximal degree [27, Theorems 7, 8]. Our reconstruction results, Theorem 2.9 for functions and Theorem 2.15 for distributions, have simpler proofs and are more general, as they are applicable to arbitrary sets of polynomials.

A further step was taken by Tuan in 70 , where he studies $L^{p}$-functions in arbitrary dimension, but also includes repeated application of some non-monomial operators, a case not considered previously by Bang. Most of the results are for $p=2$, as, e.g., Theorems 1 and 3 in [70] for the operators $\Delta$ and $\partial_{\xi}$, respectively. For general $1 \leqslant p \leqslant \infty$, he obtains [70, Theorem 4], which reads as follows: Let $P$ be a non-constant polynomial; then the Fourier transform $\mathcal{F} f$ of $f \in \mathcal{S}\left(\mathbb{R}^{d}\right)$ vanishes outside $\left\{x \in \mathbb{R}^{d}:|P(x)| \leq 1\right\}$ if, and only if,

$$
\limsup _{n \rightarrow \infty}\left\|P(i \partial)^{n} f\right\|_{p}^{1 / n} \leq 1 .
$$

This is a consequence of the stronger Theorem 2.5 in the present paper. The proof in [70, uses the Hausdorff-Young inequality, the Plancherel theorem and Hölder's inequality, and it is not obvious how this method could be adapted to yield our Theorem 2.6 for non-smooth $L^{p}$-functions for which we have, in fact, not found any previously occurring related result for non-monomial operators. For comparison we also remark that, although the statement itself is correct for arbitrary polynomials, in our opinion the proof of [70, Theorem 4] only establishes the result for polynomials which are real valued - it is not stated in [70] which polynomials are considered. The reason is that, when passing from equation (37) to (38) in [70, it is used that $P^{k}(-i \partial) \bar{f}=\overline{P^{k}(i \partial) f}$ for all $k$ and $f$, and for this one needs $P$ to be real valued. The same remark applies to the transition from equation (29) to (30) in the proof of [71, Theorem 2], while our Theorem 2.5] is stated for arbitrary non-constant polynomials: in our opinion, the proof in [71] only establishes [71, Theorem 2] for real valued polynomials. This restriction originates from the application of the Plancherel theorem in both proofs, whereas our proofs of the main results in the present paper, such as Theorem 2.5 for arbitrary polynomials, are

\footnotetext{
${ }^{3}$ There appears to be a slight mistake in the formulation of [27, Theorem 6]. In our opinion a factor $m^{N}$ should be added to the right hand side of (3.13) in [27], corresponding to the factor $n^{N}$ in our Theorem 2.2 This factor takes into account that the number of terms in the second summation on page 28 of [27] depends on $m$. There are $m^{|\nu|}$ terms, and this needs to be estimated from above by $m^{N}$.
} 
all based on the inversion theorem. This different approach avoids this limitation, which we think to be inherent to proofs based on a Plancherel theorem.

The results and approach mentioned above were in [72] and [45] generalized to the Dunkl transform, where we recall that the Dunkl transform, [37, [42, is a deformation of the Fourier transform that specializes to the Fourier transform if all the so-called root multiplicities are zero. This also means that we believe the results in 72 and 45 are valid only for real valued polynomials.

Theorem 3 in 71 is concerned with repeated application of $e^{\Delta}$ to Schwartz functions in arbitrary dimension in an $L^{p}$-context. We have no analogue of this result which, when interpreted, in our opinion gives additional evidence that some real Paley-Wiener theorems express the validity of a local spectral radius formula, a point we will argue in Section 5. In the one-dimensional case and for Schwartz functions in an $L^{p}$-context, [71, Theorem 4] involves repeated application of another operator not of the form $P(\partial)$. There is also a result for the asymptotic action of the heat semigroup on $L^{2}\left(\mathbb{R}^{d}\right)$ available; see [70, Theorem 2].

As to the complex theorems, apart from the results in Section [3, we are not aware of derivations of complex Paley-Wiener theorems from real ones, other than the results for the one-dimensional Dunkl transform in 17. The idea seems to still be very young and needs further testing. A context for a possible application is the following: In higher dimension, the results for complex Paley-Wiener theorems for the Dunkl transform in [43] and [60] seem to be not yet optimal, as the version for supports in compact convex invariant sets is thought to be true in general, but has only been proved in special cases 43 . Even though - arguing analogously to the remarks in Section 3 for the Fourier transform - an approach through real methods can only be expected to give a complex theorem for supports in a symmetric compact convex invariant set, such a version of a general complex theorem would already be a considerable improvement. With the necessary real Paley-Wiener theorem already established (the case of homogenous polynomials of degree one and $p=\infty$ of [45, Theorem 4.1]), the future may learn whether this can actually be achieved in this fashion, analogously to the one-dimensional case in [17. Similarly, the applicability of this relatively new approach to complex theorems could be investigated for transforms as in [11] and 78, where some real theorems are also already available.

\section{Perspectives and CONneCtion With LOCAL SPECTRAL TheORY}

In this section we mention some possible future developments, one of which is the interpretation of some real Paley-Wiener theorems as local spectral radius formulas.

First, as was already mentioned in Sections 1 3, and 4, we hope that the idea of deriving complex Paley-Wiener theorems from real ones will prove to be a useful one in other contexts as well, especially if the usual domain shifting is not applicable. This is indeed the case for the one-dimensional Dunkl transform, see [17, and the higher-dimensional situation can be a new testing ground.

Second, there are potential applications in support theorems for partial differential equations with constant coefficients. For example, if $f$ and $g$ are compactly supported smooth functions such that $P(\partial) f=g$, then the convex hull of the support of $f$ equals that of $g$ as follows, e.g., from the theorem of supports 40, Theorem 4.3.3]. This seems to be the best result available. However, Theorem 2.5 
gives an opportunity to obtain extra information by choosing a polynomial $Q$ and estimating

$$
\lim _{n \rightarrow \infty}\left\|Q(\partial)^{n} \mathcal{F} f(\lambda)\right\|_{p}^{1 / n}=\lim _{n \rightarrow \infty}\left\|Q(\partial)^{n} \frac{\mathcal{F} g(\lambda)}{P(i \lambda)}\right\|_{p}^{1 / n}
$$

from above, for some $1 \leq p \leq \infty$. If this limit is known to be at most $M$, then the support of $f$ must be contained in $\left\{x \in \mathbb{R}^{d}:|Q(-i x)| \leq M\right\}$. Carrying this out in a concrete situation requires a judicious choice for $Q$, probably $p=\infty$, and a skillful handling of the combinatorics. The behavior of $P(i \lambda)$ near its zero locus will also enter the computation, but for this general lower bounds are available in 61, Lemma 5.7]. Notwithstanding the complications that will arise when pursuing this idea, we mention it nevertheless, since in principle it can yield information which to our knowledge cannot be obtained otherwise.

Third, as already mentioned in Section 4, Bang also obtained an elementary version of a real Paley-Wiener theorem for the one-dimensional torus in [19. This has not been developed further, but it seems very likely that the methods of proof in the present paper can be adapted to establish real Paley-Wiener theorems for $\mathbb{R}^{d_{1}} \times \mathbb{T}^{d_{2}}$. Is it also possible to do this for Lie groups other than the connected abelian groups, with the operators $P(\partial)$ replaced by the operators in the center of the universal enveloping algebra? The answer is at least affirmative for compact connected groups [18.

Fourth, we think that the relation between real Paley-Wiener theory and local spectral theory deserves further attention, in particular the interpretation of Theorems 2.5 and 2.6 as statements belonging to that field. We will now elaborate on this, starting with bounded operators on a Banach space, where the results of our interest are already available in general, see, e.g., [44, from which the material below on the bounded case is mostly taken.

If $X$ is a Banach space, $T$ a bounded operator on $X$, and $x \in X$, then $z_{0} \in \mathbb{C}$ is said to be in the local resolvent set of $T$ at $x$, denoted by $\rho_{T}(x)$, if there is an open neighborhood $U$ of $z_{0}$ in $\mathbb{C}$, and an analytic function $\phi: U \rightarrow X$, sending $z$ to $\phi_{z}$, such that

$$
(T-z) \phi_{z}=x \quad(z \in U) .
$$

The local spectrum $\sigma_{T}(x)$ of $T$ at $x$ is the complement of $\rho_{T}(x)$ in $\mathbb{C}$. Clearly $\sigma_{T}(x) \subset \sigma(T)$, where $\sigma(T)$ is the spectrum of $T$. The operator $T$ is said to have the single-valued extension property (SVEP) if, for every non-empty open set $U \subset \mathbb{C}$, the only analytic solution $\phi: U \rightarrow X$ of the equation $(T-z) \phi_{z}=0(z \in U)$ is the zero solution. This is equivalent to requiring that the analytic local function $\phi$ in (5.1) is uniquely determined, so that one can speak of "the" local resolvent function $\phi$. For an operator $T$, having SVEP is also equivalent to 0 being the only element in $X$ with empty local spectrum [44, Proposition 1.2.16]. By [44, Proposition 1.3.2], if $T$ has SVEP, then $\sigma(T)$ is the union of all local spectra $\sigma_{T}(x)$.

The local spectral radius $r_{T}(x)$ of $T$ at $x$ is defined as

$$
r_{T}(x)=\limsup _{n \rightarrow \infty}\left\|T^{n} x\right\|^{1 / n} .
$$

If $T$ has SVEP, then by [44, Proposition 3.3.13], the local spectral radius formula

$$
r_{T}(x)=\max \left\{|z|: z \in \sigma_{T}(x)\right\} \quad(x \in X)
$$


holds, and by [81, 80, 55, the set of those $x$ in $X$ for which $\sigma_{T}(x) \neq \sigma(T)$ is then of the first category in $X$. By [44, Proposition 3.3.14], it is always true, also in the absence of SVEP, that the set of $x \in X$ for which $r_{T}(x)$ is equal to the spectral radius of $T$ is of the second category in $X$. If $T$ has Bishop's property $(\beta)$ (see 44. Definition 1.2.5] - it is immediate that property $(\beta)$ implies SVEP), then, by [4. Proposition 3.3.17], $r_{T}(x)=\lim _{n \rightarrow \infty}\left\|T^{n} x\right\|^{1 / n}$ for all $x$ in $X$, so that the local spectral radius formula (5.2) holds in a stronger form as

$$
\lim _{n \rightarrow \infty}\left\|T^{n} x\right\|^{1 / n}=\max \left\{|z|: z \in \sigma_{T}(x)\right\} \quad(x \in X) .
$$

Furthermore, a decomposable (see [44, Definition 1.1.1]) operator has property $(\beta)$ by [44, Theorem 1.2.7], and hence (5.3) holds for decomposable operators.

Now let $X=C(\Lambda)$, where $\Lambda$ is a compact Hausdorff space, and let $T$ be the operator corresponding to multiplication by a fixed function $g$. It is shown in [44. Example 1.2.11] that $T$ is decomposable and that $\sigma_{T}(f)=g(\operatorname{supp} f)$, for all $f \in C(\Lambda)$. As a consequence of the general theory above, we then know that (5.3) is valid in the form

$$
\lim _{n \rightarrow \infty}\left\|g^{n} f\right\|_{\infty}^{1 / n}=\max \{|g(\lambda)|: \lambda \in \operatorname{supp} f\} \quad(f \in C(\Lambda)) .
$$

Naturally this is also easily verified directly. The point is, however, that local spectral theory tells us without further computation that (5.4) holds once the decomposability has been established and the local spectrum has been determined.

This example makes the statement in Theorem 2.5.

$$
\lim _{n \rightarrow \infty}\left\|P(\partial)^{n} f\right\|_{p}^{1 / n}=\sup \{|P(i \lambda)|: \lambda \in \operatorname{supp} \mathcal{F} f\} \quad\left(f \in \mathcal{S}\left(\mathbb{R}^{d}\right)\right)
$$

and its analogue in Theorem 2.6 more or less plausible, since under the Fourier transform $P(\partial)$ corresponds to multiplication by $P(i \lambda)$. In fact, it raises the question as to whether Theorems 2.5 and 2.6 can conceptually be interpreted as statements in local spectral theory for unbounded operators. Are the right hand sides in these theorems the maximum modulus in the local spectrum of an unbounded operator, and is there, in addition, an a priori result available such as (5.2) or (5.3), resulting in an "explanation" of these theorems? The local spectral theory for unbounded operators is not as extensive as for the bounded case, and neither general monographs, such as [79] and 38, nor papers dedicated to local spectral theory for constant coefficient differential operators, such as [2, 3, 4, 5], contain a description of the local spectrum of an operator $P(\partial)$, or a priori results analogous to (5.2) or (5.3). The only result which is helpful in interpreting (5.5) that we are aware of is the following: If one can prove that $\{P(i \lambda): \lambda \in \operatorname{supp} \mathcal{F} f\}$ is dense in the local spectrum at $f$ of a closed operator in $L^{p}\left(\mathbb{R}^{d}\right)$ which agrees with $P(\partial)$ on $\mathcal{S}\left(\mathbb{R}^{d}\right)$, and if this set is bounded, then [79, Proposition IV.3.10] yields that

$$
\sup \{|P(i \lambda)|: \lambda \in \operatorname{supp} \mathcal{F} f\} \leq \limsup _{n \rightarrow \infty}\left\|P(\partial)^{n} f\right\|_{p}^{1 / n}<\infty .
$$

Even though this is only a partial and conditional result, it supports a conjecture that (5.5) is in reality a local spectral radius formula.

We can in fact prove this conjecture for $p=1$, and we will now proceed to do so. The definition of the local resolvent set and SVEP in the unbounded case are as above, with the additional requirement that $\phi_{z}$ is in the domain of the operator for all $z$ in $U$. 
Let $P$ be a polynomial. Consider, for $1 \leq p \leq \infty$, the associated operator $T_{p}: C_{c}^{\infty}\left(\mathbb{R}^{d}\right) \rightarrow L^{p}\left(\mathbb{R}^{d}\right)$, defined as $T_{p} f=P(\partial) f$, for $f \in C_{c}^{\infty}\left(\mathbb{R}^{d}\right)$. Then, by [59, Section 4.2], $T_{p}$ has a closed extension $\widetilde{T}_{p}$ on $L^{p}\left(\mathbb{R}^{d}\right)$, with domain $D_{\widetilde{T}_{p}}$ equal to all $f \in L^{p}\left(\mathbb{R}^{d}\right)$ such that $P(\partial) f \in L^{p}\left(\mathbb{R}^{d}\right)$, and defined as $\widetilde{T}_{p} f=P(\partial) f$ on its domain 4

Lemma 5.1. $\widetilde{T}_{1}$ has $S V E P$.

Proof. Suppose $\phi: U \rightarrow D_{\widetilde{T}_{1}}$ is analytic, and such that $\left(\widetilde{T}_{1}-z\right) \phi_{z}=0$, for all $z$ in some open non-empty $U \subset \mathbb{C}$. Taking Fourier transforms yields

$$
(P(i \lambda)-z) \mathcal{F} \phi_{z}(\lambda)=0,
$$

for all $z \in U$ and $\lambda \in \mathbb{R}^{d}$. Thus, for any fixed $\lambda \in \mathbb{R}^{d}$, we conclude that $\mathcal{F} \phi_{z}(\lambda)=$ 0 for all $z \in U$ with at most one exception, and hence, since $\mathcal{F} \phi_{z}(\lambda)$ depends continuously on $z$, we see that $\mathcal{F} \phi_{z}(\lambda)=0$ for all $z \in U$.

Note that we only needed the continuity of $\phi$ in the proof of the preceding lemma, but that it was essential that the Fourier transform of an element in the domain of $\widetilde{T}_{1}$ be a continuous function.

Although SVEP is strictly speaking not necessary for the interpretation of (5.5) as a local spectral radius formula, the following result is indispensable. Here we again use the fact that the Fourier transforms are continuous functions, but not even the continuity of $\phi$ is needed.

Lemma 5.2. If $f \in D_{\widetilde{T}_{1}}$, then

$$
\rho_{\widetilde{T}_{1}}(f) \subseteq \mathbb{C} \backslash \overline{\{P(i \lambda): \lambda \in \operatorname{supp} \mathcal{F} f\}} .
$$

Here, and in the sequel, if $A$ is a subset of the complex numbers, then $\bar{A}$ denotes its closure (and not its conjugate).

Proof. Suppose that $z_{0} \in \rho_{\widetilde{T}_{1}}(f)$, that $U$ is an open neighborhood of $z_{0}$ in $\mathbb{C}$ and that $\left(\widetilde{T}_{1}-z\right) \phi_{z}=f$ for some analytic $\phi: U \rightarrow D_{\widetilde{T}_{1}}$. Taking Fourier transforms yields $(P(i \lambda)-z) \mathcal{F} \phi_{z}(\lambda)=\mathcal{F} f(\lambda)$, for all $z \in U$ and $\lambda \in \mathbb{R}^{d}$. We conclude from this that if $\lambda_{0} \in \mathbb{R}^{d}$ is such that $P\left(i \lambda_{0}\right) \in \rho_{\widetilde{T}_{1}}(f)$, then $\mathcal{F} f\left(\lambda_{0}\right)=0$. A moment's thought shows that this implies the statement in the lemma.

For general $p$ and $f \in \mathcal{S}\left(\mathbb{R}^{d}\right)$, we can establish the reverse inclusion.

Lemma 5.3. Let $1 \leq p \leq \infty$ and $f \in \mathcal{S}\left(\mathbb{R}^{d}\right) \subseteq D_{\widetilde{T}_{p}}$. Then

$$
\rho_{\widetilde{T}_{p}}(f) \supseteq \mathbb{C} \backslash \overline{\{P(i \lambda): \lambda \in \operatorname{supp} \mathcal{F} f\}} .
$$

Proof. Suppose $z_{0} \notin \overline{\{P(i \lambda): \lambda \in \operatorname{supp} \mathcal{F} f\}}$. Then there exists an open neighborhood $U$ of $z_{0}$ in $\mathbb{C}$ such that, for each $z \in U$, the function $\psi_{z}: \mathbb{R}^{d} \rightarrow \mathbb{C}$ which is given as

$$
\psi_{z}(\lambda)= \begin{cases}\frac{\mathcal{F} f(\lambda)}{P(i \lambda)-z} & \text { if } \lambda \in \operatorname{supp} \mathcal{F} f \\ 0 & \text { if } \lambda \notin \operatorname{supp} \mathcal{F} f\end{cases}
$$

is well defined. It is then in fact in $\mathcal{S}\left(\mathbb{R}^{d}\right)$. Furthermore, it is routine to verify that the map $\psi: U \rightarrow \mathcal{S}\left(\mathbb{R}^{d}\right)$, obtained by sending $z$ to $\psi_{z}$, is analytic on $U$ when $\mathcal{S}\left(\mathbb{R}^{d}\right)$

\footnotetext{
${ }^{4}$ By [59] Theorem 4.2.1], $\widetilde{T}_{p}$ is actually the closure of $T_{p}$, if $1 \leq p<\infty$.
} 
is equipped with its usual Fréchet topology. Since the inverse Fourier transform is a homeomorphism of $\mathcal{S}\left(\mathbb{R}^{d}\right)$, and since the inclusion map of $\mathcal{S}\left(\mathbb{R}^{d}\right)$ into $L^{p}\left(\mathbb{R}^{d}\right)$ is continuous, the map $\phi: U \rightarrow D_{\widetilde{T}_{p}}$, defined as $\phi_{z}=\mathcal{F}^{-1} \psi_{z}$ for $z \in U$, is also analytic on $U$. By construction it satisfies $\left(\widetilde{T}_{p}-z\right) \phi_{z}=f$ for $z \in U$. We conclude that $z_{0} \in \rho_{\widetilde{T}_{p}}(f)$.

Combining the above lemmas, we have the following satisfactory result for $p=1$.

Corollary 5.4. The closed operator $\widetilde{T}_{1}$ on $L^{1}\left(\mathbb{R}^{d}\right)$ has SVEP. Furthermore, if $f \in \mathcal{S}\left(\mathbb{R}^{d}\right) \subseteq D_{\widetilde{T}_{1}}$, then

$$
\sigma_{\widetilde{T}_{1}}(f)=\overline{\{P(i \lambda): \lambda \in \operatorname{supp} \mathcal{F} f\}}
$$

Hence the case $p=1$ of Theorem 2.5, when read in the equivalent form

$$
\lim _{n \rightarrow \infty}\left\|\widetilde{T}_{1}^{n} f\right\|_{1}^{1 / n}=\sup \{|z|: z \in \overline{\{P(i \lambda): \lambda \in \operatorname{supp} \mathcal{F} f\}}\} \quad\left(f \in \mathcal{S}\left(\mathbb{R}^{d}\right)\right),
$$

expresses the validity of the local spectral radius formula

$$
\lim _{n \rightarrow \infty}\left\|\widetilde{T}_{1}^{n} f\right\|_{1}^{1 / n}=\sup \left\{|z|: z \in \sigma_{\widetilde{T}_{1}}(f)\right\}
$$

in the extended positive real numbers.

While establishing this result we have exploited, in particular in the proof of Lemma 5.2 , the fact that the Fourier transform maps $L^{1}\left(\mathbb{R}^{d}\right)$ into a space of distributions consisting of continuous functions. Whereas for $1<p \leq 2$ the image still consists of equivalence classes of functions, so that pointwise arguments as the above can perhaps be adapted, it is known that for $2<p \leq \infty$ the image of $L^{p}\left(\mathbb{R}^{d}\right)$ contains distributions of strictly positive order; see [40, Theorem 7.6.6] 5 Therefore it seems likely that a more refined analysis will be necessary to investigate the general case, using a priori knowledge about the elements of $D_{\widetilde{T}_{p}}$ and also the analyticity of the local resolvent functions (which we did not use in the proof of Lemma 5.2 at all) as possible extra ingredients.

In view of the above we feel confident enough to propose the following.

Conjecture 5.5. Theorems 2.5 and 2.6, when read as in (5.6), are local spectral radius formulas.

In conclusion we mention that this conjecture is also supported by the results for compact connected Lie groups in 18, where, for arbitrary $p$ and smooth functions on the group, not only the analogue of Theorem 2.5 is established, but also the interpretation, as in Corollary [5.4 of this analogous result as a local spectral radius formula.

\section{REFERENCES}

[1] L.D. Abreu, Real Paley-Wiener theorems for the Koornwinder-Swarttouw $q$-Hankel transform, J. Math. Anal. Appl. 334 (2007), 223-231. MR2332551 (2008e:33032)

[2] E. Albrecht, W.J. Ricker, Local spectral properties of constant coefficient differential operators in $L^{p}\left(R^{N}\right), J$. Operator Theory 24 (1990), 85-103. MR1086546 (92b:47048)

[3] E. Albrecht, W.J. Ricker, Functional calculi and decomposability of unbounded multiplier operators in $L^{p}\left(R^{N}\right)$, Proc. Edinburgh Math. Soc. (2) 38 (1995), 151-166. MR.1317333 (96j:47044)

\footnotetext{
${ }^{5}$ An upper bound for the order is known; see [40, p. 242].
} 
[4] E. Albrecht, W.J. Ricker, Local spectral properties of certain matrix differential operators in $L^{p}\left(R^{N}\right)^{m}$, J. Operator Theory 35 (1996), 3-37. MR1389641 (98b:47060)

[5] E. Albrecht, W.J. Ricker, On $p$-dependent local spectral properties of certain linear differential operators in $L^{p}\left(\mathbf{R}^{N}\right)$, Studia Math. 130 (1998), 23-52. MR.1623000 (99d:47018)

[6] F. Al-Musallam, A Whittaker transform over a half-line, Integral Transforms Spec. Funct. 12 (2001), 201-212. MR1872431 (2002j:44004)

[7] F. Al-Musallam, The range of finite integral transforms arising from $n$-th order singular self-adjoint differential operators, Fract. Calc. Appl. Anal. 6 (2003), 175-186. MR2035413 (2005c:44001)

[8] F. Al-Musallam, V.K. Tuan, A modified and a finite index Weber transforms, Z. Anal. Anwendungen 21 (2002), 315-334. MR1915264(2004a:44004)

[9] F. Al-Musallam, V.K. Tuan, A finite and an infinite Whittaker integral transform, Comput. Math. Appl. 46 (2003), 1847-1859. MR2018770 (2004k:44004)

[10] N.B. Andersen, On real Paley-Wiener theorems for certain integral transforms, J. Math. Anal. Appl. 288 (2003), 124-135. MR2019749 (2005e:42027)

[11] N.B. Andersen, Real Paley-Wiener theorems for the inverse Fourier transform on a Riemannian symmetric space, Pacific J. Math. 213 (2004), 1-13. MR2040247 (2005f:43007)

[12] N.B. Andersen, A simple proof of a Paley-Wiener type theorem for the Chébli-Trimèche transform, Publ. Math. Debrecen 64 (2004), 473-479. MR.2058918 (2005e:44006)

[13] N.B. Andersen, Real Paley-Wiener theorems, Bull. London Math. Soc. 36 (2004), 504-508. MR2069012 (2005g:42011)

[14] N.B. Andersen, On the range of the Chébli-Trimèche transform, Monatsh. Math. 144 (2005), 193-201. MR2130273 (2005m:44006)

[15] N.B. Andersen, Real Paley-Wiener theorems for the Hankel transform, J. Fourier Anal. Appl. 12 (2006), 17-25. MR2215674 (2006m:44001)

[16] N.B. Andersen, Real Paley-Wiener theorems for the Dunkl transform on $\mathbb{R}$, Integral Transforms Spec. Funct. 17 (2006), 543-547. MR.2246499 (2007h:43001)

[17] N.B. Andersen, M.F.E. de Jeu, Elementary proofs of Paley-Wiener theorems for the Dunkl transform on the real line, Int. Math. Res. Not. 2005, 1817-1831. MR2172939(2006h:42016)

[18] N.B. Andersen, M.F.E. de Jeu, Local spectral radius formulas on compact Lie groups, J. Lie Theory 19 (2009), 223-230.

[19] H.H. Bang, A property of infinitely differentiable functions, Proc. Amer. Math. Soc. 108 (1990), 73-76. MR:1024259 (90j:26029)

[20] H.H. Bang, Remarks on a property of infinitely differentiable functions, Bull. Polish Acad. Sci. Math. 41 (1993), 197-206. MR1414766 (97g:46025)

[21] H.H. Bang, Functions with bounded spectrum, Trans. Amer. Math. Soc. 347 (1995), 10671080. MR1283539 (95e:42008)

[22] H.H. Bang, A property of entire functions of exponential type, Analysis 15 (1995), 17-23. MR.1322126 (96c:46032)

[23] H.H. Bang, Aymptotic behaviour of the sequence of norms of derivatives, J. Math. Sci. Univ. Tokyo 2 (1995), 611-620. MR.1382522 (97i:26017)

[24] H.H. Bang, On the Bernstein-Nikolsky inequality. II., Tokyo J. Math. 18 (1995), 123-131. MR.1334710 (96d:41016)

[25] H.H. Bang, On an algebra of pseudodifferential operators, Sb. Math. 186 (1995), 929-940; translation from Mat. Sb. 186 (1995), 3-14. MR1355452 (96h:47060)

[26] H.H. Bang, The existence of a point spectral radius for pseudodifferential operators, [J] Dokl. Math. 53 (1996), 420-422; translation from Dokl. Akad. Nauk, Ross. Akad. Nauk 348 (1996), 740-742. MR1440738(98k:47101)

[27] H.H. Bang, The Paley-Wiener-Schwartz theorem for nonconvex domains, in Functional analysis and global analysis (Quezon City, 1996), 14-30, Springer, Singapore, 1997. MR1658037 (99m:46104)

[28] H.H. Bang, Paley-Wiener-Schwartz type theorems, [A] Proc. Steklov Inst. Math. 214 (1996), 291-311; translation from Tr. Mat. Inst. Steklova 214 (1997), 298-319. MR1635074 (99g:35025)

[29] H.H. Bang, Nonconvex cases of the Paley-Wiener-Schwartz theorem, Dokl. Akad. Nauk 354 (1997), 165-168. MR1471836 (98j:46037) 
[30] H.H. Bang, The study of the properties of functions belonging to an Orlicz space depending on the geometry of their spectra, Izv. Math. 61 (1997), 399-434; translation from Izv. Ross. Akad. Nauk Ser. Mat. 61 (1997), 163-198. MR1470148(99a:46047b)

[31] H.H. Bang, T.T. Mai, A property of entire functions of exponential type for Lorentz spaces, Vietnam J. Math. 32 (2004), 219-225. MR2071019 (2005f:26070)

[32] H.H. Bang, M. Morimoto, On the Bernstein-Nikolsky inequality, Tokyo J. Math. 14 (1991), 231-238. MR1108170 (92g:41020)

[33] H.H. Bang, M. Morimoto, The sequence of Luxemburg norms of derivatives, Tokyo J. Math. 17 (1994), 141-147. MR.1279574 (95b:46047)

[34] J.J. Betancor, J.D. Betancor, J.M.R. Méndez, Paley-Wiener type theorems for ChébliTrimèche transforms, Publ. Math. 60 (2002), 347-358. MR1898567 (2003b:44002)

[35] C. Chettaoui, K. Trimèche, New type Paley-Wiener theorems for the Dunkl transform on $\mathbb{R}$, Integral Transforms Spec. Funct. 14 (2003), 97-115. MR.1969838(2004d:33016)

[36] C. Chettaoui, Y. Othmani, K. Trimèche, On the range of the Dunkl transform on $\mathbb{R}$, Math. Sci. Res. J. 8 (2004), 85-103. MR2108495 (2005j:44003)

[37] C.F. Dunkl, Hankel transforms associated to finite reflection groups, in "Proceedings of the special session on hypergeometric functions on domains of positivity, Jack polynomials and applications," Tampa, 1991, Contemp. Math. 138, 123-138. MR.1199124 (94g:33011)

[38] I. Erdélyi, S. Wang, A local spectral theory for closed operators, London Mathematical Society Lecture Note Series, 105. Cambridge University Press, Cambridge, 1985. MR 817715 (87g:47059)

[39] I.M. Gelfand, G.E. Schilow, Verallgemeinerte Funktionen (Distributionen). II: Lineare topologische Räume. Räume von Grundfunktionen und verallgemeinerten Funktionen (German). Hochschulbücher für Mathematik, Bd. 48. VEB Deutscher Verlag der Wissenschaften, Berlin, 1962. MR0149275 (26:6765)

[40] L. Hörmander, The analysis of linear partial differential operators. I. Distribution theory and Fourier analysis, Second edition. Springer-Verlag, Berlin, 1990.

[41] M. Jelassi, L.T. Rachdi, On the range of the Fourier transform associated with the spherical mean operator, Fract. Calc. Appl. Anal. 7 (2004), 379-402. MR2251523 (2008b:43007)

[42] M.F.E. de Jeu, The Dunkl transform, Invent. Math. 113 (1993), 147-162. MR.1223227 (94m:22011)

[43] M. de Jeu, Paley-Wiener theorems for the Dunkl transform, Trans. Amer. Math. Soc. 358 (2006), 4225-4250. MR2231377 (2007i:33023)

[44] K.B. Laursen, M.M. Neumann, An introduction to local spectral theory, London Mathematical Society Monographs. New Series, 20. The Clarendon Press, Oxford University Press, New York, 2000. MR1747914 (2001k:47002)

[45] H. Mejjaoli, K. Trimèche, Spectrum of functions for the Dunkl transform on $R^{d}$, Fract. Calc. Appl. Anal. 10 (2007), 19-38. MR.2348864(2008i:42022)

[46] V.V. Napalkov, Convolution equations in multidimensional spaces (Russian), Nauka, Moscow, 1982. MR678923 (86g:46054)

[47] Y. Othmani, K. Trimèche, Real Paley-Wiener theorems associated with the Weinstein operator, Mediterr. J. Math. 3 (2006), 105-118. MR2215575 (2006k:42019)

[48] R. Paley, N. Wiener, The Fourier Transforms in the Complex Domain, Amer. Math. Soc. Colloq. Publ. Ser., Vol. 19, Providence, RI, 1934. MR1451142 (98a:01023)

[49] I. Pesenson, Sampling of Paley-Wiener functions on stratified groups, J. Fourier Anal. Appl. 4 (1998), 271-281. MR.1650917 (99j:41024)

[50] I. Pesenson, A sampling theorem on homogeneous manifolds, Trans. Amer. Math. Soc. 352 (2000), 4257-4269. MR1707201 (2000m:41012)

[51] I. Pesenson, Sampling of band-limited vectors, J. Fourier Anal. Appl. 7 (2001), 93-100. MR.1812998 (2002b:42034)

[52] I. Pesenson, Deconvolution of band limited functions on non-compact symmetric spaces, Houston J. Math. 32 (2006), 183-204. MR2202361 (2007c:43010)

[53] I. Pesenson, Analysis of band-limited functions on quantum graphs, Appl. Comput. Harmon. Anal. 21 (2006), 230-244. MR2259780 (2008b:81082)

[54] M. Plancherel, G. Pólya, Fonctions entières et intégrales de Fourier multiples. I, Comment. Math. Helv. 9 (1937), 224-248.

[55] B. Prunaru, M. Putinar, The generic local spectrum of any operator is the full spectrum, Bull. London Math. Soc. 31 (1999), 332-336. MR1673412 (99m:47003) 
[56] L.I. Ronkin, Introduction to the theory of entire functions of several variables. Translated from Russian by the Israel Program for Scientific Translations. Translations of Mathematical Monographs, Vol. 44. American Mathematical Society, Providence, R.I., 1974. MR 0346175 (49:10901)

[57] L.I. Ronkin, Functions of Completely Regular Growth. Translated from Russian by A. Ronkin and I. Yedvabnik. Mathematics and its Applications (Soviet Series), 81. Kluwer Academic Publishers Group, Dordrecht, 1992. MR1196691 (94d:32006)

[58] W. Rudin, Functional Analysis, McGraw-Hill Book Co., 1973. MR0365062 (51:1315)

[59] M. Schechter, Spectra of partial differential operators. North-Holland Series in Applied Mathematics and Mechanics, Vol. 14. North-Holland Publishing Co., Amsterdam-London; American Elsevier Publishing Co., Inc., New York, 1971. MR0447834 (56:6144)

[60] S. Thangavelu, Y. Xu, Convolution operator and maximal function for the Dunkl transform, J. Anal. Math. 97 (2005), 25-55. MR.2274972(2009a:43005)

[61] F. Trèves, Linear partial differential equations with constant coefficients: Existence, approximation and regularity of solutions, Mathematics and its Applications, Vol. 6. Gordon and Breach Science Publishers, New York-London-Paris, 1966. MR0224958 (37:557)

[62] F. Trèves, Topological vector spaces, distributions and kernels, Academic Press, New York London, 1967. MR0225131 (37:726)

[63] T.V. Thuong, Some properties of functions with bounded spectrum, Acta Math. Vietnam. 24 (1999), 343-352. MR1735119 (2001g:46095)

[64] V.K. Tuan, On the Paley-Wiener theorem, in Theory of Functions and Applications. Collection of Works dedicated to the Memory of M.M. Djrbashian, 193-196, Louys Publishing House, Yerevan, 1995.

[65] V.K. Tuan, Supports of functions and integral transforms, in Proceedings of International workshop on the recent advances in applied mathematics, RAAM '96, 507-521, Kuwait Univ., Department of Mathematics and Computer Science, Kuwait, 1996.

[66] V.K. Tuan, On the range of the $Y$ transform, Bull. Austral. Math. Soc. 54 (1996), 329-345. MR.1411543 (98f:44002)

[67] V.K. Tuan, On the range of the Hankel and extended Hankel transforms, J. Math. Anal. Appl. 209 (1997), 460-478. MR.1474619 (99c:44006)

[68] V.K. Tuan, Airy integral transform and the Paley-Wiener theorem, in Transform methods E special functions, Varna '96, 523-531, Bulgarian Acad. Sci., Sofia, 1998. MR.1667774 (99k:44014)

[69] V.K. Tuan, New type Paley-Wiener theorems for the modified multidimensional Mellin transform, J. Fourier Anal. Appl. 4 (1998), 317-328. MR.1650984(99k:44010)

[70] V.K. Tuan, Paley-Wiener-type theorems, Fract. Calc. Appl. Anal. 2 (1999), 135-143. MR.1689181 (2000j:42022)

[71] V.K. Tuan, On the supports of functions, Numer. Funct. Anal. Optim. 20 (1999), 387-394. MR1691371 (2000g:42012)

[72] V.K. Tuan, A real-variable Paley-Wiener theorem for the Dunkl transform, Abstract and Applied Analysis, 365-371, World Sci. Publ., River Edge, NJ, 2004. MR2095112 (2005h:44006)

[73] V.K. Tuan, Paley-Wiener and Boas theorems for singular Sturm-Liouville integral transforms, Adv. in Appl. Math. 29 (2002), 563-580. MR1943365 (2003j:47056)

[74] V.K. Tuan, A. Ismail, M. Saigo, Plancherel and Paley-Wiener theorems for an index integral transform, J. Korean Math. Soc. 37 (2000), 545-563. MR1770827 (2002g:44003)

[75] V.K. Tuan, A. Ismail, M. Saigo, On an index integral transform involving the modified Bessel function, Integral Transforms Spec. Funct. 12 (2001), 375-388. MR1872376 (2002j:44009)

[76] V.K. Tuan, A.I. Zayed, Paley-Wiener-type theorem for a class of integral transforms arising from a singular Dirac system, Z. Anal. Anwendungen 19 (2000), 695-712. MR.1784126 (2002h:42015)

[77] V.K. Tuan, A.I. Zayed, Generalization of a theorem of Boas to a class of integral transforms, Results Math. 38 (2000), 362-376. MR.1799723(2001k:44005)

[78] V.K. Tuan, A.I. Zayed, Paley-Wiener-type theorems for a class of integral transforms, J. Math. Anal. Appl. 266 (2002), 200-226. MR.1876778 (2002k:44005)

[79] F.-H. Vasilescu, Analytic functional calculus and spectral decompositions. Translated from Romanian. Mathematics and its Applications (East European Series), Vol. 1. D. Reidel Publishing Co., Dordrecht, 1982. MR690957 (85b:47016) 
[80] F.-H. Vasilescu, Analytic operators and spectral decompositions, Indiana Univ. Math. J. 34 (1985), 705-722. MR808821 (87a:47024)

[81] P. Vrbova, On local spectral properties of operators in Banach space, Czech. J. Math. 23 (1973), 483-492. MR0322536 (48:898)

Mads Clausen Institute, University of Southern Denmark, Alsion 2, DK-6400 Sønderborg, DenMark

E-mail address: byrial@mci.sdu.dk

Current address: Alssundgymnasiet Sønderborg, Grundtvigs Allé 86, 6400 Sønderborg, Denmark

E-mail address: nba@ags.dk

Mathematical Institute, Leiden University, P.O. Box 9512, 2300 RA Leiden, The NETHERLANDS

E-mail address: mdejeu@math.leidenuniv.nl 